\title{
Substituted benzimidazoles: antiviral activity and synthesis of nucleosides
}

\author{
Simone Budow, ${ }^{1}$ Mariola Kozlowska, ${ }^{2}$ Agata Gorska, ${ }^{2}$ Zygmunt Kazimierczuk, ${ }^{2}$ Henning \\ Eickmeier, ${ }^{3}$ Paolo La Colla, ${ }^{4}$ Gilles Gosselin, ${ }^{5}$ and Frank Seela ${ }^{1}$ \\ ${ }^{1}$ Laboratory of Bioorganic Chemistry and Chemical Biology, Center for Nanotechnology, \\ Heisenbergstraße 11, 48149 Münster, Germany \\ ${ }^{2}$ Institute of Chemistry, Warsaw University of Life Science, 159C Nowoursynowska St., 02-787 \\ Warsaw, Poland \\ ${ }^{3}$ Laboratorium für Chemie II, Institut für Chemie, Universität Osnabrück, Barbarastraße 7, \\ 49069 Osnabrück, Germany \\ ${ }^{4}$ Dipartimento di Science e Tecnologie Biomediche, Universtà di Cagliari, Monserrato, Cagliari, Italy \\ ${ }^{5}$ Laboratoire Coopératif Idenix-CNRS-Université Montpellier II, Montpellier, France \\ E-mail: Frank.Seela@uni-osnabrueck.de; Seela@uni-muenster.de
}

\section{Dedicated to Professor Harri Lönnberg on the occasion of his $60^{\text {th }}$ birthday}

\begin{abstract}
The antiviral activity of a series of benzimidazole derivatives and substituted benzimidazole $\beta$-Land $\beta$-D-2'-deoxyribonucleosides against selected RNA and DNA viruses including HIV-1, BVDV, YFV, DENV-2, WNV, HBV, HCV and human RSV was evaluated. In addition, the synthesis of several benzimidazole $\beta$-L-2'-deoxyribonucleosides (1-4) and 2'-deoxy-2'-fluoro- $\beta$ D-arabinofuranosyl nucleoside $\mathbf{5}$ is described. The stereoselective glycosylation of the anions of the functionalized benzimidazoles 6a, 12a and 28 with 3,5-di- $O$-(4-methylbenzoyl)-2-deoxy- $\alpha$ L-erythro-pentofuranosyl chloride (29) furnished $\beta$-L-2'-deoxyribonucleosides 1-4 while the glycosylation of the anion of 2-bromobenzimidazole (12a) with 3,5-di- $O$-benzoyl-2-deoxy-2fluoro- $\alpha$-D-arabinofuranosyl bromide 34 gave the 2'-deoxy-2-fluoro- $\beta$-D-arabinofuranosyl nucleoside 5. Moreover, the crystal structure of the benzoylated 2-bromobenzimidazole 2'deoxy-2'-fluoro- $\beta$-D-arabinofuranosyl nucleoside $\mathbf{3 5}$ is reported.
\end{abstract}

Keywords: Benzimidazoles, L-nucleosides, antiviral screening, glycosylation

\section{Introduction}

The benzimidazole system is an integral part of numerous antiparasitic, fungicidal, anthelmintic and anti-inflammatory drugs. ${ }^{1,2}$ Among them, modified 2-trifluoromethyl-benzimidazoles are 
particular promising as they show appreciable herbicidal activity due to the inhibition of photosynthesis. ${ }^{3}$ Recently, potent anticancer, antibacterial, antifungal and antiprotozoal activities were reported for halogenated 2-trifluoromethyl- and 2-pentafluoroethyl-benzimidazoles. ${ }^{4-6}$

Not only the substituted benzimidazole heterocycles themselves but also a series of modified benzimidazole nucleosides are biologically active..$^{7-9}$ Originally, their synthesis was encouraged by the discovery that 5,6-dimethyl-1-( $\alpha$-D-erythro-pentofuranosyl)-benzimidazole is a constituent of vitamin $\mathrm{B}_{12 .}{ }^{10}$ 5,6-Dichlorobenzimidazole ribonucleoside (DRB) inhibits cellular and viral RNA synthesis. ${ }^{11,12}$ However, this activity is accompanied by a substantial cytotoxicity and therefore this compound has not found application as an antiviral drug. ${ }^{13,14}$

Noteworthy is the recent finding that the L-ribonucleoside of 5,6-dichloro-2isopropylaminobenzimidazole (1263W94) shows increased activity against the herpes virus HCMV (human cytomegalovirus) in vitro compared to its parent compound 2-bromo-5,6dichloro-1- $\beta$-D-ribofuranosylbenzimidazole (BDCRB) and a low cytotoxicity. ${ }^{15}$ This is in line with the general observation that several L-nucleosides exhibit an antiviral activity comparable and sometimes greater than their D-enantiomers, due to a more favourable toxicological profile and a greater metabolic stability. ${ }^{16,17}$ Thus, various L-nucleosides have been synthesized as potential antiviral and anticancer drugs such as 3TC (lamivudine), FTC (emtricitabine) and LFMAU (clevudine). ${ }^{16-20}$ The first synthesis of a L-nucleoside ( $\beta$-L-dT) was reported in 1964 by Šmekal and Šorm ${ }^{21}$ followed by the description of $\beta$-L-adenosine by Acton, Ryan and Goodman in the same year. ${ }^{22}$ Furthermore, the anti-hepatitis B virus activity of the 2'-deoxyribonucleoside L-dT (Telbivudine, Tyzeka ${ }^{\mathrm{TM}}$, Sebivo $\left.{ }^{\mathrm{TM}}\right)^{21 \mathrm{~b}}$ against hepatitis B virus infection led to its approval as antiviral drug.

Herein, we report on the antiviral activity of benzimidazole derivatives against Flaviviridae, human immunodeficiency retrovirus (HIV-1), Hepatitis B virus (HBV), Hepatitis C virus (HCV) as well as Human Respiratory Syncytial Virus (human RSV) (Figure 1).

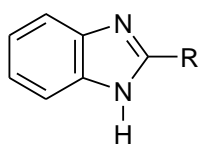

$\mathrm{R}=\mathrm{Br}$

$\mathrm{R}=\mathrm{C}_{2} \mathrm{~F}_{5}$

$\mathrm{R}=\mathrm{C}_{4} \mathrm{~F}_{9}$

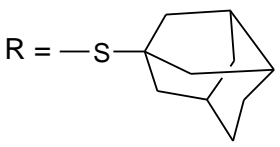

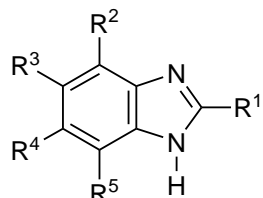

$R^{1}=C F_{3}, R^{2}=R^{5}=H, R^{3}=R^{4}=C l$

$\mathrm{R}^{1}=\mathrm{CF}_{3}, \mathrm{R}^{3}=\mathrm{R}^{5}=\mathrm{H}, \mathrm{R}^{2}=\mathrm{R}^{4}=\mathrm{Cl}$

$\mathrm{R}^{1}=\mathrm{CF}_{3}, \mathrm{R}^{2}=\mathrm{R}^{3}=\mathrm{R}^{5}=\mathrm{Br}, \mathrm{R}^{4}=\mathrm{Cl}$

$\mathrm{R}^{1}=-\mathrm{S}-\mathrm{N}^{\prime}$

$R^{2}=R^{5}=H, R^{3}=R^{4}=C l$

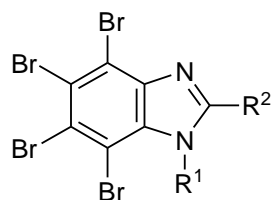

$\mathrm{R}^{1}=\mathrm{R}^{2}=\mathrm{H}$

$\mathrm{R}^{1}=\mathrm{H}, \mathrm{R}^{2}=\mathrm{C}_{3} \mathrm{~F}_{7}$

$\mathrm{R}^{1}=\mathrm{CH}_{3}, \mathrm{R}^{2}=-\mathrm{S}-\mathrm{CH}_{3}$

$\mathrm{R}^{1}=\mathrm{H}, \mathrm{R}^{2}=\underset{\mathrm{H}}{\mathrm{N}-\mathrm{CH}_{3}}$

Figure 1. Selected benzimidazole derivatives for antiviral testing.

Some of them were already described by Baczynski and Niementowski in 1902 and Büchel in 1970, respectively, ${ }^{23,24}$ others were recently reported. ${ }^{5,6,25,26}$ The introduction of side chains 
into position- 2 of halogenated or non-halogenated benzimidazoles can be achieved by different routes. ${ }^{5,6,25-27}$ One route uses non-halogenated or halogenated $o$-phenylenediamines as precursor which give after condensation with carboxylic acids and their derivatives (nitriles, imidates, orthoesters) the 2-substituted benzimidazoles (Scheme 1). In this manuscript compounds of Figure 4 were prepared employing this protocol. ${ }^{5,6}$ In another route 2,4,5,6,7-pentabrominated benzimidazole is subjected to a series of displacement reactions in position-2 using various nucleophiles (Scheme 2). ${ }^{25}$ Compounds shown in Figure 3 were synthesized according to this route.<smiles>[R][R]1ccc2nc([R])[nH]c2c1</smiles>

\section{Scheme 1}<smiles>[R]N([R])c1nc2c(Br)c(Br)c(Br)c(Br)c2n1[R]</smiles>

\section{Scheme 2}

Furthermore, a series of substituted benzimidazole $\beta$-L- and $\beta$-D-2'-deoxyribonucleosides (15) were synthesized (Figure 2). They were evaluated for their antiviral activity against Flaviviridae, HIV-1, Hepatitis B virus (HBV), Hepatitis C virus (HCV) and Human Respiratory Syncytial Virus (human RSV) and the results are discussed here.

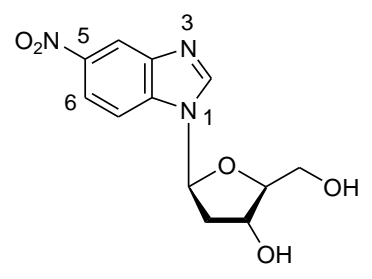

1

systematic numbering

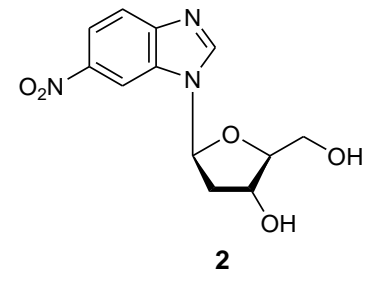

2
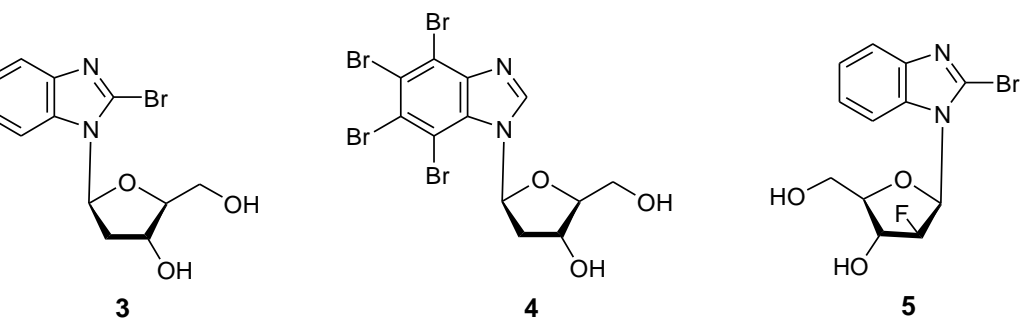

Figure 2. Substituted benzimidazole $\beta$-L- and $\beta$-D-2'-deoxyribonucleosides. 


\section{Results and Discussion}

\section{Antiviral activity and cytotoxicity}

A series of benzimidazole derivatives and benzimidazole $\beta$-L- and $\beta$-D-2'-deoxyribonucleosides were evaluated in cell-based assays by determining the $50 \%$ cytotoxicity concentration $\left(\mathrm{CC}_{50}\right)$ and the $50 \%$ inhibitory concentration $\left(\mathrm{EC}_{50}\right)$ as previously described. ${ }^{28}$ Among the tested viruses, representatives of three genera of the Flaviviridae which belong to the class of positive-sense single-stranded RNA (ssRNA ${ }^{+}$) viruses were selected. These are Pestivirus (bovine viral diarrhoea virus, BVDV), Flavivirus including yellow fever virus (YFV), dengue virus type 2 (DENV-2) and West Nile virus (WNV) and Hepacivirus (hepatitis C virus; HCV). Moreover, activity against the representative member of the Retroviridae (ssRNA ${ }^{+}$) the human immunodeficiency retrovirus (HIV-1) and the DNA virus Hepatitis B (HBV) was evaluated as well as the activity against the negative-sense single-stranded RNA virus (ssRNA-) Human Respiratory Syncytial Virus (human RSV). The data are summarized in Tables 1-7 and the structures of compounds 1-27 are given in Figures 3-6.

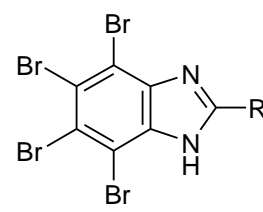

6a-u

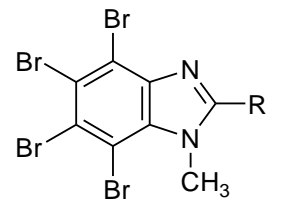

7c-d, j, k, v-w

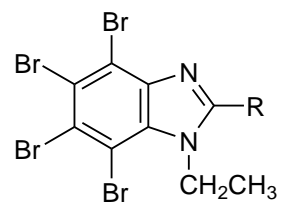

$8 d$

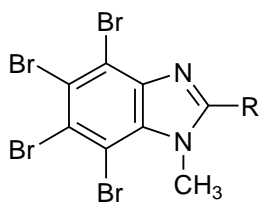

9n<smiles>O=c1[nH]c2c(Br)c(Br)c(Br)c(Br)c2n1P</smiles>

10: $\mathrm{R}=\mathrm{H}$ 11: $\mathrm{R}=\mathrm{CH}_{3}$

a: $R=H, \mathbf{b}: R=C l, \mathbf{c}: R=B r, \mathbf{d}: R=\mathrm{CF}_{3}, \mathbf{e}: R=\mathrm{C}_{2} \mathrm{~F}_{5}, \mathbf{f}: \mathrm{R}=\mathrm{C}_{3} \mathrm{~F}_{7}, \mathbf{g}: \mathrm{R}=\mathrm{C}_{4} \mathrm{~F}_{9}, \mathbf{h}: \mathrm{R}=\mathrm{NH}_{2}$

i: $\mathrm{R}=-\stackrel{\mathrm{H}}{\mathrm{N}}-\mathrm{CH}_{3}$

$\mathrm{j}: \mathrm{R}=-\mathrm{N}\left(\mathrm{CH}_{3}\right)_{2}$

$\mathbf{k}: \mathrm{R}=\stackrel{\mathrm{H}}{\mathrm{N}}<$

$\mathrm{I}: \mathrm{R}=-\mathrm{H}^{\mathrm{H}}-\mathrm{N}^{\prime}$

$0: R=-s-\overbrace{}^{\mathrm{OH}}$

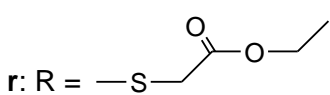

$\mathrm{R}: \mathrm{R}=-\mathrm{C}$ $\mathbf{m}: \mathrm{R}=\mathrm{SH}$<smiles>OCC(O)CSC#P</smiles><smiles>O=[N+]([S-])C=CCc1ccc([N+](=O)[O-])cc1</smiles>

$\mathrm{v}: \mathrm{R}=-\mathrm{O}<$ n: $\mathrm{R}=-\mathrm{S}-\mathrm{CH}_{3}$<smiles></smiles>

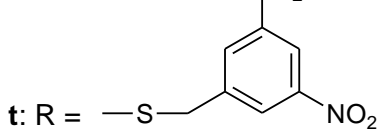

$\mathbf{w}: \mathrm{R}=-\mathrm{O}-$

Figure 3. 4,5,6,7-Tetra-brominated benzimidazoles for antiviral testing. 
Table 1. Antiviral activity of 4,5,6,7-tetrabrominated benzimidazoles against selected ssRNA ${ }^{+}$ viruses (part 1)

\begin{tabular}{|c|c|c|c|c|c|c|c|c|c|c|}
\hline \multirow[b]{2}{*}{ Comp. } & \multicolumn{2}{|c|}{$\mathrm{HIV}_{-1}{ }^{\mathrm{a}}$} & \multicolumn{2}{|c|}{$\mathrm{BVDV}^{\mathrm{b}}$} & \multicolumn{2}{|c|}{$\mathrm{YFV}^{\mathrm{c}}$} & \multicolumn{2}{|c|}{ DENV-2 ${ }^{\mathrm{c}}$} & \multicolumn{2}{|c|}{$\mathrm{WNV}^{\mathrm{c}}$} \\
\hline & $\mathrm{CC}_{50} \mathrm{~d}^{\mathrm{d}}$ & $\mathrm{EC}_{50}{ }^{\mathrm{e}}$ & $\mathrm{CC}_{50}{ }^{\mathrm{f}}$ & $\mathrm{EC}_{50}{ }^{\mathrm{g}}$ & $\mathrm{CC}_{50}{ }^{\mathrm{h}}$ & $\mathrm{EC}_{50}{ }^{\mathrm{i}}$ & $\mathrm{CC}_{50}^{\mathrm{h}}$ & $\mathrm{EC}_{50}{ }^{\mathrm{i}}$ & $\mathrm{CC}_{50}{ }^{\mathrm{h}}$ & $\mathrm{EC}_{50}{ }^{\mathrm{i}}$ \\
\hline $6 \mathbf{a}$ & 15 & $>15$ & 13 & $>13$ & 49 & 33 & $>100$ & $>100$ & $>100$ & $>100$ \\
\hline $6 b$ & 8.0 & $>8.0$ & 3.2 & $>3.2$ & 3.0 & $>3.0$ & 3.0 & $>3.0$ & 3.0 & $>3.0$ \\
\hline $6 c$ & 9.0 & $>9.0$ & 3.8 & $>3.8$ & 2.6 & $>2.6$ & 2.0 & $>2.0$ & 2.0 & $>2.0$ \\
\hline $6 d$ & $<3.7$ & n.d. & 2 & $>2$ & 1 & $>1$ & 1 & $>1$ & n.d. & $>1$ \\
\hline $6 e$ & $<3.7$ & n.d. & 0.8 & $>0.8$ & 0.6 & $>0.6$ & 0.5 & $>0.5$ & n.d. & $>0.5$ \\
\hline $6 f$ & 1.0 & $>1.0$ & 2.0 & $>2.0$ & 0.8 & $>0.8$ & 0.6 & $>0.6$ & 0.6 & $>0.6$ \\
\hline $6 g$ & 1.2 & $>1.2$ & $<3.7$ & n.d. & $<3.7$ & n.d. & $<3.7$ & n.d. & $>3.7$ & n.d. \\
\hline $6 h$ & 4.0 & $>4.0$ & 4.3 & $>4.3$ & 58 & 41 & 66 & 46 & 66 & 31 \\
\hline $6 \mathbf{i}$ & 7.0 & $>7.0$ & 65 & $>65$ & $>100$ & $>100$ & $>100$ & $>100$ & $>100$ & $>100$ \\
\hline $6 \mathbf{j}$ & 17 & $>17$ & $>100$ & $>100$ & $>100$ & $>100$ & $>100$ & $>100$ & $>100$ & $>100$ \\
\hline $6 k$ & 4.0 & $>4.0$ & $>100$ & $>100$ & $>100$ & $>100$ & $>100$ & $>100$ & $>100$ & $>100$ \\
\hline 61 & 5.0 & $>5.0$ & 24 & $>24$ & 56 & 23 & 18 & $>18$ & 18 & $>18$ \\
\hline $6 \mathrm{~m}$ & 65 & $>65$ & $>100$ & $>100$ & $>100$ & $>100$ & $>100$ & $>100$ & $>100$ & $>100$ \\
\hline $6 n$ & 7.0 & $>7.0$ & 15 & $>15$ & $>100$ & $>100$ & $>100$ & $>100$ & $>100$ & $>100$ \\
\hline 60 & 15 & $>15$ & $>100$ & $>100$ & $>100$ & $>100$ & $>100$ & $>100$ & $>100$ & $>100$ \\
\hline $6 p$ & 17 & 7.0 & 14 & $>14$ & 25 & $>25$ & $>100$ & $>100$ & $>100$ & $>100$ \\
\hline $6 q$ & $>100$ & $>100$ & 61 & $>61$ & 39 & $>39$ & 49 & $>49$ & 49 & $>49$ \\
\hline $6 r$ & $\geq 100$ & $>100$ & $>100$ & $>100$ & $>100$ & $>100$ & $>100$ & $>100$ & $>100$ & $>100$ \\
\hline $6 s$ & 4.0 & $>4.0$ & 18 & $>18$ & 3.0 & $>3.0$ & 4.0 & $>4.0$ & 4.0 & $>4.0$ \\
\hline $6 t$ & 3.7 & $>3.7$ & 27 & $>27$ & 4.0 & $>4.0$ & 7.0 & $>7.0$ & 7.0 & $>7.0$ \\
\hline $6 u$ & 3.0 & $>3.0$ & 6.0 & $>6.0$ & 3.0 & $>3.0$ & 5.0 & $>5.0$ & 5.0 & $>5.0$ \\
\hline $7 c$ & $\geq 100$ & $>100$ & $>100$ & $>100$ & $>100$ & $>100$ & $>100$ & $>100$ & $>100$ & $>100$ \\
\hline $7 d$ & $>100$ & $>100$ & $>100$ & $>100$ & $>100$ & $>100$ & $>100$ & $>100$ & $>100$ & $>100$ \\
\hline $7 \mathbf{j}$ & $\geq 100$ & $>100$ & $>100$ & $>100$ & $>100$ & $>100$ & $>100$ & $>100$ & $>100$ & $>100$ \\
\hline $7 k$ & 68 & $>68$ & $>100$ & $>100$ & $>100$ & $>100$ & $>100$ & $>100$ & $>100$ & $>100$ \\
\hline $7 v$ & 76 & $>76$ & $>100$ & $>100$ & $>100$ & $>100$ & $>100$ & $>100$ & $>100$ & $>100$ \\
\hline $7 w$ & $>100$ & $>100$ & $>100$ & $>100$ & $>100$ & $>100$ & $>100$ & $>100$ & $>100$ & $>100$ \\
\hline $8 d$ & 80 & $>80$ & $>100$ & $>100$ & $>100$ & $>100$ & $>100$ & $>100$ & $>100$ & $>100$ \\
\hline $9 n$ & $\geq 100$ & $>100$ & $>100$ & $>100$ & $>100$ & $>100$ & $>100$ & $>100$ & $>100$ & $>100$ \\
\hline 10 & $\geq 100$ & $>100$ & 77 & $>77$ & $>100$ & $>100$ & $>100$ & $>100$ & $>100$ & $>100$ \\
\hline 11 & $\geq 100$ & $>100$ & $>100$ & $>100$ & $>100$ & $>100$ & $>100$ & $>100$ & $>100$ & $>100$ \\
\hline
\end{tabular}

${ }^{\mathrm{a}}$ Retroviridae. ${ }^{\mathrm{b}}$ Pestivirus. ${ }^{\mathrm{c}}$ Flavivirus. ${ }^{\mathrm{d}}$ Compound concentration $(\mu \mathrm{M})$ required to reduce MT$4\left(\mathrm{CD}^{+}\right.$human T-cells containing an integrated HTLV-1 genome) cell proliferation by $50 \%$, as determined by the 3-(4,5-dimethylthiazol-2-yl)-2,5-diphenyl-tetrazolium bromide (MTT) method, under conditions that allow untreated controls to undergo at least three consecutive 
rounds of multiplication. ${ }^{\mathrm{e}}$ Compound concentration $(\mu \mathrm{M})$ required to achieve $50 \%$ protection of MT-4 cell lines from virus-induced cytopathogenicity, as determined by the MTT method. ${ }^{\mathrm{f}}$ Compound concentration $(\mu \mathrm{M})$ required to reduce the viability of mock-infected Madin Darby Bovin Kidney (MDBK) cells by 50\%, as determined by the MTT method. ${ }^{g}$ Compound concentration $(\mu \mathrm{M})$ required to achieve $50 \%$ protection of preinfected MDBK cell lines from virus-induced cytopathogenicity, as determined by the MTT method. ${ }^{\mathrm{h}}$ Compound concentration $(\mu \mathrm{M})$ required to reduce the viability of mock-infected baby hamster kidney (BHK) cells by $50 \%$, as determined by the MTT method. ${ }^{\mathrm{i}}$ Compound concentration $(\mu \mathrm{M})$ required to reduce the virus plaque number by $50 \%$ in BHK cell lines. n.d. not yet determined.

In the series of 4,5,6,7-tetrabrominated benzimidazole derivatives (Figure 3) no compound showing a significant selective activity against one of the ssRNA ${ }^{+}$viruses HIV-1, BVDV, YFV, DENV-2 or WNV could be identified as shown in Table 1. Among them, compounds $\mathbf{6 h}, \mathbf{6 l}$ and 6p exhibit a certain antiviral activity against HIV-1, YFV, DENV-2 or WNV. However, the antiviral activity is accompanied by cytotoxicity for the host cell lines within the same concentration range.

The closely related 4,5,6,7-tetrabromobenzimidazole derivatives 6d-g having a trifluoromethyl, pentafluoroethyl, heptafluoropropyl or nonafluorobutyl side chain attached to position-2 of the benzimidazole ring exhibit strong cytotoxicity against the host cell lines in the low micromolar range $(0.5-2.0 \mu \mathrm{M})$. On the other hand compounds $\mathbf{7 d}$ and $\mathbf{7 w}$ are completely inactive against all the tested ssRNA ${ }^{+}$viruses and are also not cytotoxic. 
<smiles>[R]c1nc2ccccc2[nH]1</smiles>

$12 a, c-e, q$<smiles>[R]c1nc2cc(Br)ccc2[nH]1</smiles>

$15 b$<smiles>[R]c1nc2cc(Cl)c(Cl)cc2[nH]1</smiles>

13b, d-h, j, I, o-q<smiles>[R]c1nc2c(Cl)cc(Cl)cc2[nH]1</smiles>

$14 b, d, f-j, I, r$<smiles>[R7]c1nc2c([R])c([R])c([R7])c([R7])c2[nH]1</smiles><smiles>[R]c1nc2c(Br)cc(Br)cc2[nH]1</smiles>

16b-c, f-j, l-n, r<smiles>[R]c1nc2cc(C(=O)O)ccc2[nH]1</smiles>

17g, k-I, p, s
18: $R^{1}=C_{3}, R^{2}=R^{3}=R^{4}=B r, R^{5}=H$

19: $R^{1}=C F_{3}, R^{3}=R^{4}=B r, R^{2}=R^{5}=H$

20: $R^{1}=C F_{3}, R^{2}=R^{4}=R^{5}=H, R^{3}=C l$

21: $R^{1}=C_{3}, R^{2}=R^{5}=B r, R^{3}=R^{4}=C l$

22: $R^{1}=C_{3}, R^{2}=R^{3}=R^{5}=B r, R^{4}=C l$

23: $R^{1}=C_{3}, R^{2}=R^{4}=C l, R^{3}=R^{5}=B r$

a: $R=B r, b: R=C F_{3}, \mathbf{c}: R=C_{2} F_{5}, d: R=C_{3} F_{7}, \mathbf{e}: R=C_{4} F_{9}$

$\mathrm{f}: \mathrm{R}=-\mathrm{S}-\mathrm{N}^{\prime} \times \mathrm{HCl}$<smiles>[X]C1CCCCN1CCSC#I</smiles><smiles>O=[N+]([O-])c1ccc(CSC#P)cc1</smiles><smiles>O=PCSCc1ccc([N+](=O)[O-])cc1[N+](=O)[O-]</smiles>

$\mathbf{r}: \mathrm{R}=-\mathrm{S}-\mathrm{OH}$<smiles>[X]CN(CC)CCSC#[Hg]</smiles><smiles>[X]CN(C)CCCSC=P</smiles><smiles>[X][CH]Cl</smiles><smiles>[R]C#SCc1ccccc1</smiles><smiles>O=[N+]([O-])c1cc(CSC#P)cc([N+](=O)[O-])c1</smiles><smiles>C=Nc1cc([N+](=O)[O-])ccc1CSP</smiles><smiles>[R]#SCSc1ccc(Cl)c(Cl)c1</smiles>

s: $\mathrm{R}=-\mathrm{s} \longrightarrow$

Figure 4. Halogenated and other benzimidazole derivatives for antiviral testing. 
Table 2. Antiviral activity of halogenated benzimidazoles derivatives against selected ssRNA ${ }^{+}$ viruses (part 2) $)^{\mathrm{j}}$

\begin{tabular}{|c|c|c|c|c|c|c|c|c|c|c|}
\hline \multirow[b]{2}{*}{ Comp. } & \multicolumn{2}{|c|}{ HIV- $1^{\mathrm{a}}$} & \multicolumn{2}{|c|}{$\mathrm{BVDV}^{\mathrm{b}}$} & \multicolumn{2}{|c|}{$\mathrm{YFV}^{\mathrm{c}}$} & \multicolumn{2}{|c|}{ DENV-2 ${ }^{\mathrm{c}}$} & \multicolumn{2}{|c|}{$\mathrm{WNV}^{\mathrm{c}}$} \\
\hline & $\mathrm{CC}_{50} \mathrm{~d}^{\mathrm{d}}$ & $\mathrm{EC}_{50} \mathrm{e}^{\mathrm{e}}$ & $\mathrm{CC}_{50}{ }^{\mathrm{f}}$ & $\mathrm{EC}_{50}{ }^{\mathrm{g}}$ & $\mathrm{CC}_{50}{ }^{\mathrm{h}}$ & $\mathrm{EC}_{50}{ }^{\mathrm{i}}$ & $\mathrm{CC}_{50}{ }^{\mathrm{h}}$ & $\mathrm{EC}_{50}{ }^{\mathrm{i}}$ & $\mathrm{CC}_{50}{ }^{\mathrm{h}}$ & $\mathrm{EC}_{50}{ }^{\mathrm{i}}$ \\
\hline $12 a$ & $>100$ & $>100$ & $>100$ & $>100$ & $>100$ & $>100$ & $>100$ & $>100$ & $>100$ & $>100$ \\
\hline $12 \mathrm{c}$ & $>100$ & $>100$ & $>100$ & $>100$ & $>100$ & $>100$ & $>100$ & $>100$ & $>100$ & $>100$ \\
\hline 12d & $>100$ & $>100$ & $>100$ & $>100$ & $>100$ & $>100$ & $>100$ & $>100$ & $>100$ & $>100$ \\
\hline $12 \mathrm{e}$ & 44 & $>44$ & $>100$ & $>100$ & $>100$ & $>100$ & $>100$ & $>100$ & $>100$ & $>100$ \\
\hline $12 q$ & $>100$ & 64 & $>100$ & $>100$ & $>100$ & $>100$ & $>100$ & $>100$ & $>100$ & $>100$ \\
\hline $13 b$ & 4.0 & $>4.0$ & 3.0 & $>3.0$ & 3.5 & $>3.5$ & 12 & $>12$ & 12 & $>12$ \\
\hline 13d & 9 & $>9$ & 2.5 & $>2.5$ & 2.3 & $>2.3$ & 1.3 & $>1.3$ & 1.3 & $>1.3$ \\
\hline $13 e$ & 9 & $>9$ & 11 & $>>11$ & 9.5 & $>9.5$ & 1 & $>1$ & 1 & $>1$ \\
\hline $13 f$ & $>100$ & $>100$ & $>100$ & $>100$ & $>100$ & $>100$ & $>100$ & $>100$ & $>100$ & $>100$ \\
\hline $13 g$ & $>100$ & $>100$ & $>100$ & 100 & 100 & 29 & $\geq 100$ & $\geq 100$ & $>100$ & $>100$ \\
\hline $13 \mathrm{~h}$ & 19 & $>19$ & 8.8 & $>8.8$ & 18 & $>18$ & 49 & $>49$ & 49 & $>49$ \\
\hline $13 \mathbf{j}$ & $>100$ & $>100$ & $>100$ & $>100$ & $\geq 100$ & $>100$ & 47 & $>47$ & 47 & $>47$ \\
\hline 131 & 70 & $>70$ & 37 & $>37$ & 52 & $\geq 25$ & 100 & $>100$ & 100 & $>100$ \\
\hline 130 & 18 & $>18$ & 70 & $>70$ & $\geq 100$ & $>100$ & $>100$ & $>100$ & $>100$ & $>100$ \\
\hline $13 p$ & $>100$ & $>100$ & 65 & $>65$ & 100 & $>100$ & 59 & $>59$ & 59 & $>59$ \\
\hline $13 q$ & 38 & $>38$ & $>100$ & $>100$ & $>100$ & $>100$ & $>100$ & $>100$ & $>100$ & $>100$ \\
\hline $14 \mathrm{~b}$ & 10 & $>10$ & 4.2 & $>4.2$ & 5.0 & $>5.0$ & 4.2 & $>4.2$ & 4.2 & $>4.2$ \\
\hline $14 d$ & 2.6 & $>2.6$ & 2.9 & $>2.9$ & 2.7 & $>2.7$ & 2.4 & $>2.4$ & 2.4 & $>2.4$ \\
\hline $14 f$ & 55 & $>55$ & 72 & 32 & 35 & $>35$ & 33 & 19 & 33 & $>33$ \\
\hline $14 \mathrm{~g}$ & 22 & $>22$ & 48 & $>48$ & 26 & $>26$ & 31 & $>31$ & 31 & $>31$ \\
\hline $14 \mathrm{~h}$ & 52 & $>52$ & 44 & 32 & 20 & $>20$ & 11 & 86 & 11 & 11 \\
\hline $14 i$ & 25 & $>25$ & 50 & $>50$ & 51 & $>51$ & 54 & $>54$ & 54 & $>54$ \\
\hline $14 \mathbf{j}$ & 70 & $>70$ & $>100$ & $>100$ & $>100$ & $>100$ & $>100$ & $>100$ & $>100$ & $>100$ \\
\hline $15 b$ & 9 & $>9$ & 5.5 & $>5.5$ & 11 & $>11$ & 15 & $>15$ & 15 & $>15$ \\
\hline $16 \mathrm{~b}$ & 17 & $>17$ & 3.7 & $>3.7$ & 5.5 & $>5.5$ & 4.6 & $>4.6$ & 4.6 & $>4.6$ \\
\hline $16 c$ & 6 & $>6$ & 3.3 & $>3.3$ & 3.3 & $>3.3$ & 2.3 & $>2.3$ & 2.3 & $>2.3$ \\
\hline $16 f$ & 47 & $>47$ & 60 & 16 & 16 & $\geq 16$ & 49 & $\geq 6.6$ & 49 & $>49$ \\
\hline $16 \mathrm{~g}$ & 16 & $>16$ & 35 & 18 & 18 & $>18$ & 57 & 24 & 57 & $>57$ \\
\hline $16 \mathrm{~h}$ & 20 & $>20$ & 70 & $>70$ & 45 & $>45$ & 30 & $>30$ & 30 & $>30$ \\
\hline $16 i$ & 15 & $>15$ & 44 & $>44$ & 54 & $>54$ & $>100$ & $>100$ & $>100$ & $>100$ \\
\hline $16 j$ & 68 & $>68$ & $>100$ & $>100$ & $>100$ & $>100$ & $>100$ & $>100$ & $>100$ & $>100$ \\
\hline 161 & 16 & $>16$ & 12 & $>12$ & 9 & $>9$ & 8 & $>8$ & 8 & $>8$ \\
\hline $16 \mathrm{~m}$ & 7 & $>7$ & 14.5 & 10 & 17 & 9.5 & 20 & $>20$ & 20 & $>20$ \\
\hline $16 n$ & 18 & $>18$ & $>100$ & $>100$ & $>100$ & $>100$ & $>100$ & $>100$ & $>100$ & $>100$ \\
\hline 160 & 7.3 & $>7.3$ & 14 & 10 & 17 & 9 & 20 & $>20$ & 20 & $>20$ \\
\hline $16 r$ & 83 & $>83$ & 100 & $>100$ & 79 & $>79$ & $>100$ & $>100$ & $>100$ & $>100$ \\
\hline
\end{tabular}


Table 2. Continued

\begin{tabular}{|c|c|c|c|c|c|c|c|c|c|c|}
\hline \multirow[b]{2}{*}{ Comp. } & \multicolumn{2}{|c|}{ HIV- $1^{\text {a }}$} & \multicolumn{2}{|c|}{$\mathrm{BVDV}^{\mathrm{b}}$} & \multicolumn{2}{|c|}{$\mathrm{YFV}^{\mathrm{c}}$} & \multicolumn{2}{|c|}{ DENV-2 ${ }^{\mathrm{c}}$} & \multicolumn{2}{|c|}{$\mathrm{WNV}^{\mathrm{c}}$} \\
\hline & $\mathrm{CC}_{50}{ }^{\mathrm{d}}$ & $\mathrm{EC}_{50}{ }^{\mathrm{e}}$ & $\mathrm{CC}_{50}{ }^{\mathrm{f}}$ & $\mathrm{EC}_{50}{ }^{\mathrm{g}}$ & $\mathrm{CC}_{50}{ }^{\mathrm{h}}$ & $\mathrm{EC}_{50}{ }^{\mathrm{i}}$ & $\mathrm{CC}_{50}{ }^{\mathrm{h}}$ & $\mathrm{EC}_{50}{ }^{\mathrm{i}}$ & $\mathrm{CC}_{50}{ }^{\mathrm{h}}$ & $\mathrm{EC}_{50}{ }^{\mathrm{i}}$ \\
\hline $17 \mathrm{~g}$ & $>100$ & $>100$ & $>100$ & $>100$ & $>100$ & $>100$ & $>100$ & $>100$ & $>100$ & $>100$ \\
\hline $17 k$ & $>100$ & $>100$ & $>100$ & $>100$ & $>100$ & $>100$ & $>100$ & $>100$ & $>100$ & $>100$ \\
\hline 171 & $>100$ & $>100$ & $>100$ & $>100$ & $>100$ & $>100$ & $>100$ & $>100$ & $>100$ & $>100$ \\
\hline $17 p$ & $>100$ & $>100$ & 99 & $>99$ & 96 & $>96$ & 83 & $>83$ & 83 & $>83$ \\
\hline $17 \mathrm{~s}$ & $>100$ & $>100$ & $>100$ & $>100$ & $>100$ & $>100$ & $>100$ & $>100$ & $>100$ & $>100$ \\
\hline 18 & 3.0 & $>3.0$ & 1.3 & $>1.3$ & 1.0 & $>1.0$ & 2.3 & $>2.3$ & 2.3 & $>2.3$ \\
\hline 19 & 4.0 & $>4.0$ & 2.0 & $>2.0$ & 2.3 & $>2.3$ & 4.0 & $>4.0$ & 4.0 & $>4.0$ \\
\hline 20 & 18 & $>18$ & 13 & $>13$ & 30 & $>30$ & 34 & $>34$ & 34 & $>34$ \\
\hline 21 & 4.0 & $>4.0$ & 2.7 & $>2.7$ & 1.7 & $>1.7$ & 2.3 & $>2.3$ & 2.3 & $>2.3$ \\
\hline 22 & 4.0 & $>4.0$ & 3.5 & $>3.5$ & 1.8 & $>1.8$ & 2.0 & $>2.0$ & 2.0 & $>2.0$ \\
\hline 23 & 4.0 & $>4.0$ & 3.8 & $>3.8$ & 2.0 & $>2.0$ & 2.0 & $>2.0$ & 2.0 & $>2.0$ \\
\hline
\end{tabular}

${ }^{\mathrm{a}}$ Retroviridae. ${ }^{\mathrm{b}}$ Pestivirus. ${ }^{\mathrm{c}}$ Flavivirus. ${ }^{\mathrm{j}}$ For details of the antiviral assays see Table 1.

Next, various non-halogenated, chlorinated and brominated benzimidazole derivatives as well as 2-carboxybenzimidazoles (Figure 4) were evaluated for their antiviral activity against ssRNA $^{+}$viruses (HIV-1, BVDV, YFV, DENV-2, WNV). Unfortunately, the compounds of this series do not show selective antiviral activity against any of the tested ssRNA ${ }^{+}$viruses. The moderate antiviral activities of $\mathbf{1 3 g}, \mathbf{1 4 f}, \mathbf{1 6 f}, \mathbf{1 6 g}, \mathbf{1 6 m}$ and $\mathbf{1 6 0}$ are accompanied by cytotoxicity against the host cell lines within the same concentration range as it was also observed for the 4,5,6,7-tetra-brominated benzimidazoles shown above.

However, from the data given in Table 2 it can be seen that compounds 14d, 16c, 18, 19 and 21-23 are strongly cytotoxic within a range of 1.0-4.0 $\mu \mathrm{M}$ in all tested cell lines whereas compounds 12a, 12c, 12d, 13f, $17 \mathrm{~g}, 17 \mathrm{k}$ and $17 \mathrm{l}$ are completely inactive against all tested ssRNA $^{+}$viruses and are also non-cytotoxic. 
<smiles>[R]c1nc2cc([N+](=O)[O-])ccc2[nH]1</smiles>

24g, $h$<smiles>[R7]c1nc2cc([N+](=O)[O-])c([N+](=O)[O-])cc2n1[R7]</smiles>

25a-f

a: $\mathrm{R}^{1}=\mathrm{H}, \mathrm{R}^{2}=\square \mathrm{OH}: \mathrm{R}^{1}=\mathrm{H}, \mathrm{R}^{2}=\square \mathrm{N}\left(\mathrm{CH}_{3}\right)_{2} \times \mathrm{xHCl}$

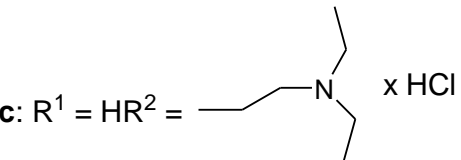

d: $\mathrm{R}^{1}=\mathrm{H}, \mathrm{R}^{2}=\mathrm{N}^{\mathrm{N}\left(\mathrm{CH}_{3}\right)_{2}} \times \mathrm{HCl}$

e: $R^{1}=H, R^{2}=$

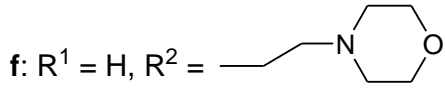

$\mathbf{h}: \mathrm{R}=-\mathrm{S}$

Figure 5. Nitrobenzimidazole derivatives for antiviral testing.

Table 3. Antiviral activity of nitrobenzimidazole derivatives against selected ssRNA ${ }^{+}$viruses $(\text { part } 3)^{\mathrm{j}}$

\begin{tabular}{|c|c|c|c|c|c|c|c|c|c|c|}
\hline \multirow[b]{2}{*}{ Comp. } & \multicolumn{2}{|c|}{ HIV-1 ${ }^{\text {a }}$} & \multicolumn{2}{|c|}{$\mathrm{BVDV}^{\mathrm{b}}$} & \multicolumn{2}{|c|}{$\mathrm{YFV}^{\mathrm{c}}$} & \multicolumn{2}{|c|}{ DENV-2 ${ }^{\mathrm{c}}$} & \multicolumn{2}{|c|}{$\mathrm{WNV}^{\mathrm{c}}$} \\
\hline & $\mathrm{CC}_{50}{ }^{\mathrm{d}}$ & $\mathrm{EC}_{50}{ }^{\mathrm{e}}$ & $\mathrm{CC}_{50}{ }^{\mathrm{f}}$ & $\mathrm{EC}_{50}{ }^{\mathrm{g}}$ & $\mathrm{CC}_{50}{ }^{\mathrm{h}}$ & $\mathrm{EC}_{50}{ }^{\mathrm{i}}$ & $\mathrm{CC}_{50}{ }^{\mathrm{h}}$ & $\mathrm{EC}_{50}{ }^{\mathrm{i}}$ & $\mathrm{CC}_{50}{ }^{\mathrm{h}}$ & $\mathrm{EC}_{50}^{\mathrm{i}}$ \\
\hline $24 \mathrm{~g}$ & 15 & $>15$ & $>100$ & $>100$ & $>100$ & $>100$ & $>100$ & $>100$ & $>100$ & $>100$ \\
\hline $24 \mathrm{~h}$ & 13 & $>13$ & 9 & $>9$ & 12 & $>12$ & 19 & $>19$ & $>19$ & $>19$ \\
\hline $25 a$ & $>100$ & $>100$ & $>100$ & $>100$ & $>100$ & $>100$ & $>100$ & $>100$ & $>100$ & $>100$ \\
\hline $25 b$ & $>100$ & $>100$ & $>100$ & $>100$ & $>100$ & $>100$ & $>100$ & $>100$ & $>100$ & $>100$ \\
\hline $25 c$ & $>100$ & $>100$ & $>100$ & $>100$ & $>100$ & $>100$ & $>100$ & $>100$ & $>100$ & $>100$ \\
\hline 25d & $>100$ & $>100$ & $>100$ & $>100$ & $>100$ & $>100$ & $>100$ & $>100$ & $>100$ & $>100$ \\
\hline $25 \mathrm{e}$ & $>100$ & $>100$ & $>100$ & $>100$ & $>100$ & $>100$ & $>100$ & $>100$ & $>100$ & $>100$ \\
\hline $25 f$ & $>100$ & $>100$ & $>100$ & $>100$ & $>100$ & $>100$ & $>100$ & $>100$ & $>100$ & $>100$ \\
\hline
\end{tabular}

${ }^{\mathrm{a}}$ Retroviridae. ${ }^{\mathrm{b}}$ Pestivirus. ${ }^{\mathrm{c}}$ Flavivirus. ${ }^{\mathrm{j}}$ For details of the antiviral assays see Table 1.

The data summarized in Table 3 show that none of the nitrobenzimidazole derivatives (Figure 5) exhibit selective antiviral activity against ssRNA ${ }^{+}$viruses. Compound $\mathbf{2 4 g}$ is cytotoxic against the cell line used in the HIV-1 assay while compound $\mathbf{2 4 h}$ is toxic against all tested cell lines. 


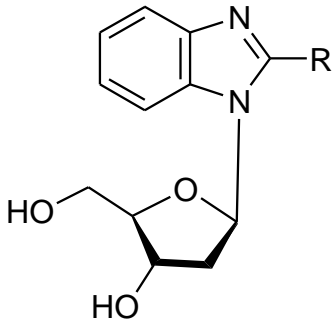

26a: $R=B r$

b: $\mathrm{R}=\mathrm{H}$

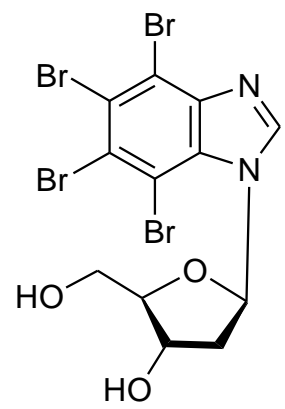

27

Figure 6. Benzimidazole $\beta$-D-2'-deoxyribonucleosides selected for antiviral testing.

Table 4. Antiviral activity of benzimidazole $\beta$-L- and $\beta$-D-2'-deoxyribonucleosides against selected ssRNA ${ }^{+}$viruses $(\text {part } 4)^{\mathrm{j}}$

\begin{tabular}{|c|c|c|c|c|c|c|c|c|c|c|}
\hline \multirow[b]{2}{*}{ Comp. } & \multicolumn{2}{|c|}{ HIV- $1^{\text {a }}$} & \multicolumn{2}{|c|}{$\mathrm{BVDV}^{\mathrm{b}}$} & \multicolumn{2}{|c|}{$\mathrm{YFV}^{\mathrm{c}}$} & \multicolumn{2}{|c|}{ DENV-2 ${ }^{\mathrm{c}}$} & \multicolumn{2}{|c|}{$\mathrm{WNV}^{\mathrm{c}}$} \\
\hline & $\mathrm{CC}_{50}{ }^{\mathrm{d}}$ & $\mathrm{EC}_{50}{ }^{\mathrm{e}}$ & $\mathrm{CC}_{50}{ }^{\mathrm{f}}$ & $\mathrm{EC}_{50}{ }^{\mathrm{g}}$ & $\mathrm{CC}_{50} \mathrm{~h}^{\mathrm{h}}$ & $\mathrm{EC}_{50} \mathrm{i}^{\mathrm{i}}$ & $\mathrm{CC}_{50}{ }^{\mathrm{h}}$ & $\mathrm{EC}_{50}{ }^{\mathrm{i}}$ & $\mathrm{CC}_{50} \mathrm{~h}^{\mathrm{h}}$ & $\mathrm{EC}_{50}{ }^{\mathrm{i}}$ \\
\hline 1 & n.d. & n.d. & $>100$ & $>100$ & $>100$ & $>100$ & $>100$ & $>100$ & n.d. & $>100$ \\
\hline 2 & n.d. & n.d. & $>100$ & $>100$ & $>100$ & $>100$ & $>100$ & $>100$ & n.d. & $>100$ \\
\hline 3 & $>100$ & $>100$ & $>100$ & $>100$ & $>100$ & $>100$ & $>100$ & $>100$ & n.d. & $>100$ \\
\hline 4 & 7.9 & $>7.9$ & 5 & $>5$ & 5 & $>5$ & 6.5 & $>6.5$ & 6.5 & $>6.5$ \\
\hline 5 & $>100$ & $>100$ & $>100$ & $>100$ & $>100$ & $>100$ & $>100$ & $>100$ & $>100$ & $>100$ \\
\hline $26 a$ & $>100$ & $>100$ & $>100$ & $>100$ & $>100$ & $>100$ & $>100$ & $>100$ & $>100$ & $>100$ \\
\hline 27 & 4.0 & $>4.0$ & 4 & 4 & 5.7 & $>5.7$ & 45 & 24 & 45 & 18 \\
\hline
\end{tabular}

${ }^{\mathrm{a}}$ Retroviridae. ${ }^{\mathrm{b}}$ Pestivirus. ${ }^{\mathrm{c}}$ Flavivirus. ${ }^{\mathrm{j}}$ For details of the antiviral assays see Table 1. n.d. not yet determined.

Then, the benzimidazole $\beta$-L-2'-deoxyribonucleosides 1-4 as well as the fluoroarabino nucleoside 5 (Figure 2) and closely related $\beta$-D-2'-deoxyribonucleosides (26a, 27; Figure 6) were screened for their antiviral activity against ssRNA ${ }^{+}$viruses as demonstrated by Table $4 .^{29}$ Among them, the 4,5,6,7-tetrabrominated benzimidazole $\beta$-L-2'-deoxyribonucleoside 4 as well as its corresponding $\beta$-D-enantiomer 27 show similar antiviral activity against the tested ssRNA ${ }^{+}$ viruses. However, these compounds are also cytotoxic against the host cell lines within the same concentration range. For comparison, 4,5,6,7-tetrabromobenzimidazole 6a also exhibits antiviral activity accompanied by toxicity (see Table 1). The 4,5,6,7-tetrabrominated benzimidazole 2'deoxyribonucleosides $\mathbf{4}$ and $\mathbf{2 7}$ are more toxic than the heterocycle $\mathbf{6 a}$ itself. It can be assumed that the nucleosides can enter the cells more efficiently and function as a release system delivering the active benzimidazole derivative 6a. Unfortunately, the other benzimidazole L- and D-nucleosides show no antiviral activity and are non-toxic. 
Table 5. Activity of benzimidazole derivatives against HBV

\begin{tabular}{|c|c|c|c|c|c|c|c|}
\hline & & $\mathrm{HBV}^{\mathrm{a}}$ & HBV $^{\mathrm{a}}$ & & & $\mathrm{HBV}^{\mathrm{a}}$ & $\mathrm{HBV}^{\mathrm{a}}$ \\
\hline Comp. & $\mathrm{CC}_{50}{ }^{\mathrm{b}}$ & $\mathrm{EC}_{50} \mathrm{c}$ & $\mathrm{EC}_{50} \mathrm{~d}^{\mathrm{d}}$ & Comp. & $\mathrm{CC}_{50}{ }^{\mathrm{b}}$ & $\mathrm{EC}_{50}{ }^{\mathrm{c}}$ & $\mathrm{EC}_{50}{ }^{\mathrm{d}}$ \\
\hline 1 & $>100$ & $>10$ & $>10$ & $13 e$ & 1.8 & $>1$ & $>1$ \\
\hline 2 & $>100$ & $>10$ & $>10$ & $13 f$ & $>100$ & $>10$ & $>10$ \\
\hline 3 & $>100$ & $>10$ & $>10$ & $13 \mathrm{~g}$ & 58 & $>10$ & $>10$ \\
\hline 4 & 7.8 & $>10$ & $>10$ & $13 \mathbf{j}$ & 64 & $>10$ & $>10$ \\
\hline 5 & $>50$ & $>10$ & $>10$ & $13 I$ & 17 & $>10$ & $>10$ \\
\hline $6 a$ & 9.0 & 0.3 & 0.5 & $13 p$ & 5 & $>5$ & $>5$ \\
\hline $\mathbf{6 b}$ & 4.5 & $>10$ & $>10$ & $14 \mathrm{~b}$ & 12 & $>10$ & $>10$ \\
\hline $6 c$ & 4.3 & $>10$ & $>10$ & $14 d$ & 2.0 & $>2.0$ & $>2.0$ \\
\hline 6d & 5 & $>1$ & $>1$ & $14 \mathrm{~h}$ & 58 & $>10$ & $>10$ \\
\hline $6 e$ & 3 & $>1$ & $>1$ & $15 \mathrm{~b}$ & 27 & $>1$ & $>1$ \\
\hline 6f & 0.8 & $>0.8$ & $>0.8$ & $16 \mathrm{~b}$ & 17 & $>1$ & $>1$ \\
\hline $6 \mathrm{~h}$ & $<3.7$ & 0.3 & 0.3 & $16 \mathrm{c}$ & 15 & $>1$ & $>1$ \\
\hline $6 \mathrm{i}$ & 6.6 & 0.5 & 0.2 & $16 f$ & 30 & $>10$ & $>10$ \\
\hline $6 \mathbf{j}$ & 100 & 5.0 & 1.0 & $16 \mathrm{~g}$ & 19 & $>10$ & $>10$ \\
\hline $6 k$ & 5.8 & 0.7 & 0.4 & $16 \mathrm{~m}$ & 14 & $>10$ & $>10$ \\
\hline 61 & 6.0 & $>10$ & $>10$ & $16 n$ & 15 & $>10$ & $>10$ \\
\hline $6 \mathrm{~m}$ & n.d. & 0.5 & 1.0 & 160 & 14 & $>10$ & $>10$ \\
\hline $6 n$ & 12 & $>10$ & $>10$ & $17 \mathrm{~g}$ & $>100$ & $>10$ & $>10$ \\
\hline 60 & 9.0 & 0.4 & 0.6 & $17 \mathbf{k}$ & $>100$ & $>10$ & $>10$ \\
\hline $6 q$ & $>100$ & $>10$ & $>10$ & 171 & $>100$ & $>10$ & $>10$ \\
\hline $6 r$ & $>100$ & $>10$ & $>10$ & $17 p$ & $>100$ & $>10$ & $>10$ \\
\hline $6 \mathrm{~s}$ & 3.0 & $>3.0$ & $>3.0$ & $17 \mathrm{~s}$ & $>100$ & $>10$ & $>10$ \\
\hline $6 t$ & 3.6 & $>3.7$ & $>3.7$ & 18 & 2.7 & $>2.7$ & $>2.7$ \\
\hline $6 u$ & n.d. & $>10$ & $>10$ & 19 & 2.8 & $>2.8$ & $>2.8$ \\
\hline $7 c$ & 33 & 10 & 10 & 20 & 14 & $>10$ & $>10$ \\
\hline $7 d$ & $>100$ & $>10$ & $>10$ & 21 & 3.6 & $>10$ & $>10$ \\
\hline $7 \mathbf{j}$ & $>100$ & 0.8 & 0.6 & 22 & 3.7 & $>3.0$ & $>3.0$ \\
\hline $7 k$ & $>100$ & $>10$ & $>10$ & 23 & 3.7 & $>7.5$ & $>7.5$ \\
\hline $7 v$ & $>100$ & 10 & 10 & $25 a$ & $>100$ & $>10$ & $>10$ \\
\hline $7 w$ & $>100$ & $>10$ & $>10$ & $25 b$ & $>100$ & $>10$ & $>10$ \\
\hline $8 d$ & $>100$ & $>10$ & $>10$ & $25 \mathrm{c}$ & $>100$ & $>10$ & $>10$ \\
\hline $9 n$ & 100 & $>10$ & $>10$ & $25 d$ & $>100$ & $>10$ & $>10$ \\
\hline 10 & n.d. & $>10$ & $>10$ & $25 \mathrm{e}$ & $>100$ & $>10$ & $>10$ \\
\hline
\end{tabular}


Table 5. Continued

\begin{tabular}{|c|c|c|c|c|c|c|c|}
\hline & & $\begin{array}{c}\mathrm{HBV}^{\mathrm{a}} \\
\mathrm{RI}\end{array}$ & $\begin{array}{c}\mathrm{HBV}^{\mathrm{a}} \\
\text { Vir }\end{array}$ & & & $\begin{array}{c}\mathrm{HBV}^{\mathrm{a}} \\
\mathrm{RI} \\
\end{array}$ & $\begin{array}{c}\mathrm{HBV}^{\mathrm{a}} \\
\mathrm{Vir} \\
\end{array}$ \\
\hline Comp. & $\mathrm{CC}_{50}{ }^{\mathrm{b}}$ & $\mathrm{EC}_{50}{ }^{\mathrm{c}}$ & $\mathrm{EC}_{50}{ }^{\mathrm{d}}$ & Comp. & $\mathrm{CC}_{50}{ }^{\mathrm{b}}$ & $\mathrm{EC}_{50}{ }^{\mathrm{c}}$ & $\mathrm{EC}_{50}{ }^{\mathrm{d}}$ \\
\hline 11 & $>100$ & $>10$ & $>10$ & $25 f$ & $>100$ & $>10$ & $>10$ \\
\hline $12 \mathrm{a}$ & $>100$ & $>10$ & $>10$ & 26 & $>100$ & $>10$ & $>10$ \\
\hline $12 \mathrm{e}$ & 52 & $>10$ & $>10$ & 27 & 4.7 & $>10$ & $>10$ \\
\hline $13 b$ & $\leq 3$ & $>3.0$ & $>3.0$ & 3TC & & 0.04 & 0.025 \\
\hline 13d & $>3.0$ & $>1$ & $>1$ & & & & \\
\hline
\end{tabular}

a Hepadnaviridae. ${ }^{\mathrm{b}}$ Compound concentration $(\mu \mathrm{M})$ required to reduce the viability of heptatocellular carcinoma (HepG2) cells by $50 \%$, as determined by the MTT method. ${ }^{c}$ Compound concentration $(\mu \mathrm{M})$ required to reduce the intracellular HBV DNA (HBV replicative intermediates, RI). ${ }^{d}$ Compound concentration $(\mu \mathrm{M})$ required to reduce the intracellular HBV virion (HBV Vir).

Several benzimidazole derivatives were also tested for their activity against the Hepatitis B virus (HBV) which belongs to the DNA viruses. In this assay the $50 \%$ inhibitory concentration $\left(\mathrm{EC}_{50}\right)$ was determined for the virion (Vir) and the replicative intermediate (RI) of HBV. Table 5 shows that within the tested compounds the 4,5,6,7-tetrabrominated benzimidazole derivatives $\mathbf{6 j}$ and $7 \mathbf{j}$ both carrying a dimethylamino side chain attached to position- 2 of the heterocycle show anti-HBV activity against the virion and the replicative intermediate in a low micromolar concentration range $\left(\mathbf{6 j}: \mathrm{EC}_{50}(\mathrm{RI})=5.0 \mu \mathrm{M}, \mathrm{EC}_{50}(\mathrm{Vir})=1.0 \mu \mathrm{M}\right.$ and $\mathbf{7 j}: \mathrm{EC}_{50}(\mathrm{RI})=0.8 \mu \mathrm{M}$, $\left.\mathrm{EC}_{50}(\mathrm{Vir})=0.6 \mu \mathrm{M}\right)$ while the cytotoxicity is less pronounced $\left(\mathbf{6 j} \mathbf{\mathbf { j }} \mathrm{CC}_{50}=100 \mu \mathrm{M}\right.$ and $\mathbf{7 j}$ : $\mathrm{CC}_{50}$ $>100 \mu \mathrm{M})$. Interestingly, among the 4,5,6,7-tetrabrominated benzimidazoles having different side chains attached to position-2 (Figure 3) several compounds $(\mathbf{6 a}, \mathbf{h}, \mathbf{i}, \mathbf{k}, \mathbf{o})$ are toxic in low concentration $(<1 \mu \mathrm{M})$. Apart from the L-nucleoside $\mathbf{4}$ which is cytotoxic the tested nucleosides 1-3, 5 are without activity. 
Table 6. Activity of benzimidazole derivatives in the $\mathrm{HCV}^{\mathrm{a}}$ replicon system

\begin{tabular}{|c|c|c|c|c|c|}
\hline \multirow[b]{2}{*}{ Compound } & \multicolumn{2}{|c|}{ HCV Replicon } & \multirow[b]{2}{*}{ Compound } & \multicolumn{2}{|c|}{ HCV Replicon } \\
\hline & $\%$ INHIB $^{\mathrm{b}}$ & $\% \mathrm{TOX}^{\mathrm{c}}$ & & $\%$ INHIB $^{\mathrm{b}}$ & $\% \mathrm{TOX}^{\mathrm{c}}$ \\
\hline $6 a$ & 47.4 & 1.6 & $7 w$ & 10.6 & 0 \\
\hline $6 c$ & 29.9 & 0 & $8 d$ & 0 & 9.0 \\
\hline $6 h$ & 78.6 & 42.5 & 11 & 0 & 0 \\
\hline $6 \mathbf{i}$ & 24.5 & 42.0 & $12 \mathbf{a}$ & 0 & 1.6 \\
\hline $\mathbf{6 j}$ & 0 & 77.3 & $12 \mathrm{e}$ & 21.9 & 0 \\
\hline $6 \mathbf{k}$ & 0 & 33.6 & $13 b$ & 66.6 & 68.0 \\
\hline 60 & 48.5 & 10.0 & $14 \mathrm{~b}$ & 15.0 & 28.0 \\
\hline $6 q$ & 7.2 & 9.0 & $16 \mathrm{~g}$ & 7.3 & 14.1 \\
\hline $6 r$ & 20.5 & 56.0 & $16 n$ & 10.4 & 19.0 \\
\hline $7 c$ & 89.4 & 4.3 & 18 & 63.0 & 63.5 \\
\hline 7d & 0 & 0 & 20 & 22.3 & 48.0 \\
\hline $7 \mathbf{j}$ & 0 & 2.4 & 21 & 63.1 & 69.6 \\
\hline $7 \mathbf{k}$ & 0 & 0 & 23 & 53.8 & 69.0 \\
\hline
\end{tabular}

${ }^{a}$ Hepacivirus. ${ }^{\mathrm{b}}$ The observed inhibition of HCV replicon replication at the test concentration of $15 \mu \mathrm{M}$ of drug as determined by the HCV replicon (ELISA) assay. ${ }^{\mathrm{c}}$ The observed cellular toxicity at the test concentration of $15 \mu \mathrm{M}$ of drug.

Regarding the activity in the HCV replicon system a series of benzimidazoles were selected for testing (Table 6). Their ability to inhibit the HCV replicon replication at the test concentration of $15 \mu \mathrm{M}$ was evaluated by an HCV replicon assay (ELISA) as previously described. ${ }^{28}$ The cellular toxicity was determined at the test concentration of $15 \mu \mathrm{M}$ of drug. ${ }^{28}$ For most compounds inhibition of the HCV replicon replication is accompanied by cytotoxicity. Only in case of $\mathbf{6 c}, \mathbf{7 w}$ and $\mathbf{1 2 e}$ inhibition was observed without cytotoxicity. 
Table 7. Activity of benzimidazole derivatives against $\mathrm{RSV}^{\mathrm{a}}$

\begin{tabular}{|c|c|c|c|c|c|c|c|c|}
\hline \multirow[b]{2}{*}{ Comp. } & \multicolumn{2}{|c|}{ RSV } & \multirow[b]{2}{*}{ Comp. } & \multicolumn{2}{|c|}{ RSV } & \multirow[b]{2}{*}{ Comp. } & \multicolumn{2}{|c|}{ RSV } \\
\hline & $\mathrm{CC}_{50} \mathrm{~b}^{\mathrm{b}}$ & $\mathrm{EC}_{50}{ }^{\mathrm{c}}$ & & $\mathrm{EC}_{50} \mathrm{~b}$ & $\mathrm{EC}_{50}{ }^{\mathrm{c}}$ & & $\mathrm{EC}_{50} \mathrm{~b}^{\mathrm{b}}$ & $\mathrm{EC}_{50^{\mathrm{c}}}$ \\
\hline 3 & $>100$ & $>100$ & $6 q$ & 100 & $>100$ & $13 b$ & 3.4 & $>3.4$ \\
\hline 4 & 5 & $>5.0$ & $6 r$ & $\geq 100$ & $>100$ & $14 b$ & 1.7 & $>1.7$ \\
\hline 5 & $>100$ & $>100$ & $6 s$ & 3.5 & $>3.5$ & $14 \mathrm{~h}$ & 36 & $>36$ \\
\hline $6 a$ & 2.3 & $>2.3$ & $6 t$ & 10 & $>10$ & $14 d$ & 1.0 & $>1.0$ \\
\hline $6 b$ & 0.5 & $>0.5$ & $6 u$ & 2.7 & $>2.7$ & $16 \mathrm{~g}$ & $>100$ & $>100$ \\
\hline $6 c$ & 1.3 & $>1.3$ & $7 c$ & $>100$ & $>100$ & $16 n$ & 1.1 & $>1.1$ \\
\hline $6 f$ & 0.6 & $>0.6$ & $7 d$ & $>100$ & $>100$ & 160 & 6.0 & $>6.0$ \\
\hline $6 h$ & 1.0 & $>1.0$ & $7 \mathbf{j}$ & $>100$ & $>100$ & 18 & 0.8 & $>0.8$ \\
\hline $6 \mathbf{i}$ & 5.0 & $>5.0$ & $7 k$ & $>100$ & $>100$ & 19 & 1.2 & $>1.2$ \\
\hline $\mathbf{6 j}$ & $>100$ & $>100$ & $7 w$ & $>100$ & $>100$ & 20 & 20 & $>20$ \\
\hline $6 k$ & $>100$ & $>100$ & $8 d$ & $>100$ & $>100$ & 21 & 0.7 & $>0.7$ \\
\hline 61 & 6.0 & $>6.0$ & $9 n$ & $>100$ & $>100$ & 22 & 0.7 & $>0.7$ \\
\hline $6 \mathrm{~m}$ & $>100$ & $>100$ & 10 & 100 & $>100$ & 23 & 0.8 & $>0.8$ \\
\hline $6 n$ & 6.0 & $>6.0$ & 11 & $\geq 100$ & $>100$ & 26 & $>100$ & $>100$ \\
\hline 60 & 23 & $>23$ & $12 \mathrm{a}$ & $>100$ & $>100$ & 27 & 2.0 & $>2.0$ \\
\hline $6 p$ & 72 & $>72$ & $12 \mathrm{e}$ & $>100$ & $>100$ & & & \\
\hline
\end{tabular}

${ }^{\mathrm{a}}$ Paramyxoviridae. ${ }^{\mathrm{b}}$ Compound concentration $(\mu \mathrm{M})$ required to reduce the Vero cell number determined by methylene blue staining. ${ }^{c}$ Compound concentration $(\mu \mathrm{M})$ required to reduce the virus plaque number by $50 \%$ in Vero cell lines.

The Respiratory Syncytial Virus (RSV) is a major cause of respiratory illness in infants and young children. However, antiviral approaches are still required to treat this infection. From Table 7 it can be seen that none of the tested benzimidazole derivatives show selective antiviral activity against RSV. The compounds are either toxic or completely inactive. However, in the series of the halogenated benzimidazoles including the 4,5,6,7-tetrabrominated benzimidazole Lnucleoside $\mathbf{4}$ most compounds are cytotoxic.

\section{Nucleoside Synthesis}

\section{Synthesis of benzimidazole $\beta$-L-2'-deoxyribonucleosides}

Considering the identical properties of L- and D-nucleosides and their precursors in a non-chiral environment, the protocols developed for the chemical synthesis of their D-counterparts can be employed for the preparation for the L-enantiomers. ${ }^{30}$ Instead of 3,5-di-O-(4-methylbenzoyl)-2deoxy- $\alpha$-D-erythro-pentofuranosyl chloride the corresponding L-enantiomer is used as sugar component. 3,5-Di- $O$-(4-methylbenzoyl)-2-deoxy- $\alpha$-L-erythro-pentofuranosyl chloride (29) was already described in $1964{ }^{21}$ and was prepared according to the procedure for the corresponding D-halogenose. ${ }^{31}$ 
The glycosylation of 5(6)-nitrobenzimidazole ${ }^{32}$ (28) with the $\alpha$-L-2-deoxyribofuranosyl halide 29 was performed in $\mathrm{MeCN}$ in the presence of powdered $\mathrm{KOH}$ using TDA-1 (tris[2-(2methoxyethoxy)ethyl]amine) as phase-transfer catalyst (nucleobase anion conditions ${ }^{33}$ ) (Scheme 3). The glycosylation led to a mixture of the $\mathrm{N}^{1}$ - and $\mathrm{N}^{3}$-glycosylated regioisomers $\mathbf{3 0}$ and 31 which were separated by flash chromatography. The faster migrating compound was isolated in $26 \%$ (30) and the slower one (31) in 32\% yield. Both compounds were deprotected with $1 \mathrm{M}$ $\mathrm{NaOMe} / \mathrm{MeOH}$. Crystallization from methanol afforded the corresponding regioisomeric nucleosides 1 and $\mathbf{2}$ as colourless crystals in 59\% and 62\% yield, respectively.

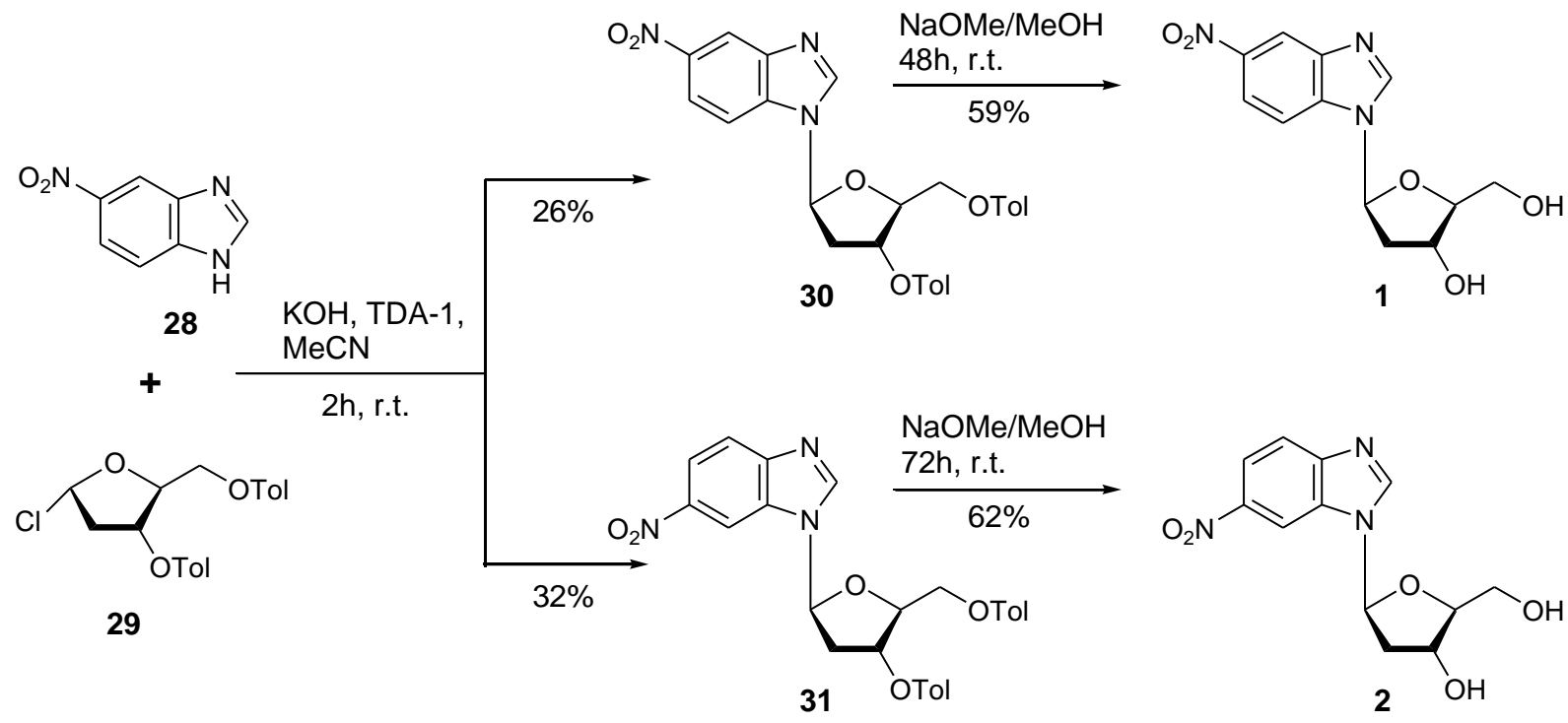

\section{Scheme 3}

2-Bromobenzimidazole ${ }^{25}(\mathbf{1 2 a})$ and 4,5,6,7-tetrabromobenzimidazole ${ }^{23}$ (6a) were prepared as described recently. Glycosylation of the brominated benzimidazoles 12a or $6 \mathbf{a}$ with halogenose 29 was achieved by employing the sodium salt glycosylation procedure as shown in Scheme 4. The sugar component 29 was added to a solution of 2-bromobenzimidazole 12a or 4,5,6,7tetrabromobenzimidazole $\mathbf{6 a}$ in anhydrous $\mathrm{MeCN}$ in the presence of $\mathrm{NaH}$. The glycosylation reaction afforded the toluoyl-protected $\beta$-L-nucleosides 32 or 33 in $63 \%$ and $51 \%$ yield, respectively. Deprotection of both nucleosides with $1 \mathrm{M} \mathrm{NaOMe/MeOH}$ furnished the brominated benzimidazole $\beta$-L-nucleosides 3 (88\%) and 4 (51\%). 
<smiles>[R2]c1[nH]c2c([R])nc=2c([R2])c([R2])c1[R2]</smiles>

12a: $R_{1}=B r ; R_{2}=H$

6a: $R_{1}=H ; R_{2}=B r$

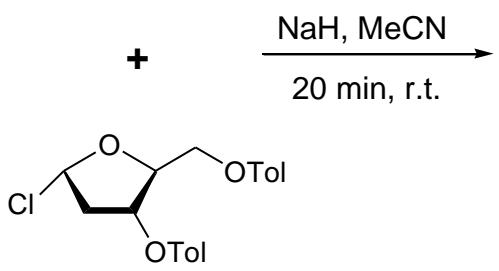

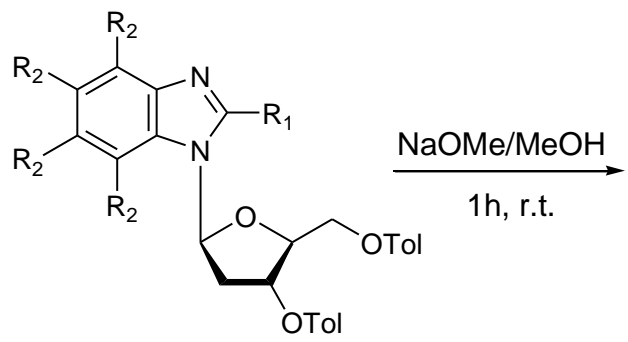

32: $R_{1}=B r ; R_{2}=H ; 63 \%$

33: $R_{1}=H ; R_{2}=B r ; 51 \%$

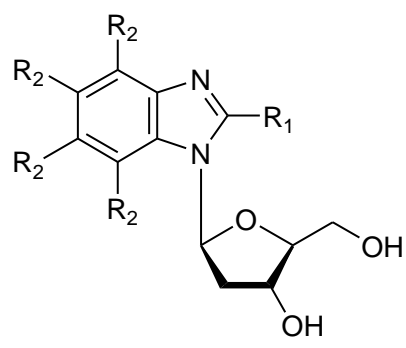

3: $\mathrm{R}_{1}=\mathrm{Br} ; \mathrm{R}_{2}=\mathrm{H} ; 88 \%$

4: $R_{1}=H ; R_{2}=B r ; 51 \%$

\section{Scheme 4}

Synthesis of 2-bromo-1-[2-deoxy-2-fluoro- $\beta$-D-arabinofuranosyl]-benzimidazole (5). The convergent synthesis of a number of 2'-deoxy-2-fluoro- $\beta$-D-arabinofuranosyl nucleosides have already been reported. ${ }^{34}$ The preparation of 2-bromo-2'-deoxy-2'-fluoro- $\beta$-Darabinofuranosylbenzimidazole (5) started with the known 3,5-di- $O$-benzoyl-2-deoxy-2-fluoro- $\alpha$ D-arabinofuranosyl bromide 34 which was obtained from the commercially available 1-O-acetyl$2,3,5$-tri- $O$-benzoyl- $\beta$-D-ribofuranose by a three step procedure. ${ }^{35}$

The glycosylation reaction of nucleobase 12a with fluoro sugar 34 was performed by nucleobase anion glycosylation (MeCN/KOH/TDA-1) in a similar way as described for the nitrobenzimidazole $\beta$-L-nucleosides above (Scheme 5). This reaction afforded the protected 2'deoxy-2'-fluoro nucleoside 35 in $42 \%$ yield. Deprotection of 35 with methanolic ammonia resulted in the removal of the benzoyl groups and gave the free nucleoside $\mathbf{5}$ in $61 \%$.
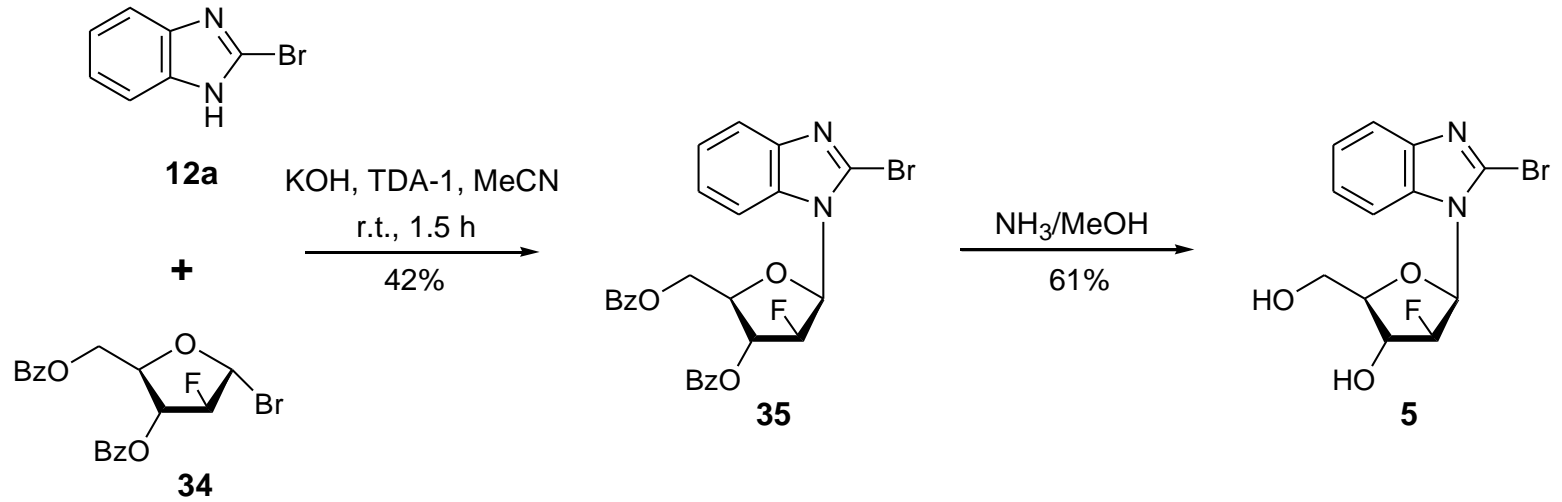

\section{Scheme 5}


Spectroscopic data and single crystal X-ray analysis. Compounds 1-5 as well as their intermediates were characterized by ${ }^{1} \mathrm{H}$ NMR spectra (experimental section) and ${ }^{13} \mathrm{C}-\mathrm{NMR}$ spectra (Table 8) as well as by elemental analysis. For the benzimidazole $\beta$-L-2'deoxyribonucleosides 1-2 and 30-31 the assignment of the ${ }^{13} \mathrm{C}-\mathrm{NMR}$ chemical shifts were made according to the corresponding D-nucleosides. ${ }^{36}$

For compounds 4, 33 the ${ }^{13} \mathrm{C}-\mathrm{NMR}$ signals of the functionalized benzimidazole nucleobases were assigned according to ${ }^{13} \mathrm{C}-\mathrm{NMR}$ chemical shifts of the non-brominated benzimidazole nucleoside (26b) (Figure 6). ${ }^{37}$ Moreover, gated decoupled ${ }^{1} \mathrm{H}^{13} \mathrm{C}$ NMR spectra were measured to confirm the assignment of the ${ }^{13} \mathrm{C}$-signals of nucleosides $\mathbf{5}, \mathbf{3 2}$ and $\mathbf{3 5}$ (Table 9).

For the 2-bromobenzimidazole 2'-deoxy-2'-fluoro- $\beta$-D-arabinofuranosyl nucleosides 35 and 5 the presence of the fluorine atome in the sugar moiety was confirmed by ${ }^{19} \mathrm{~F}-\mathrm{NMR}$ spectra (35: -194.2 ppm and 5: -194.0 ppm). Moreover, $J_{\mathrm{C}, \mathrm{F}}$ coupling constants are given in Table 9.

Table 8. ${ }^{13} \mathrm{C}$ NMR chemical shifts of benzimidazole $\beta$-L- and $\beta$-D-2'-deoxyribonucleosides ${ }^{\text {a }}$

\begin{tabular}{llllllll}
\hline & $\mathrm{C}(2)^{\mathrm{b}}$ & $\mathrm{C}(3 \mathrm{a})^{\mathrm{b}}$ & $\mathrm{C}(4)^{\mathrm{b}}$ & $\mathrm{C}(5)^{\mathrm{b}}$ & $\mathrm{C}(6)^{\mathrm{b}}$ & $\mathrm{C}(7)^{\mathrm{b}}$ & $\mathrm{C}(7 \mathrm{a})^{\mathrm{b}}$ \\
\hline $\mathbf{1}$ & 146.1 & 143.0 & 115.7 & $137.4^{\mathrm{d}}$ & 118.2 & 112.1 & 143.0 \\
$\mathbf{2}$ & 147.2 & 143.0 & 119.9 & 117.6 & $132.2^{\mathrm{d}}$ & 108.8 & 148.2 \\
$\mathbf{3}$ & 129.2 & 143.2 & 118.8 & $123.2^{\mathrm{d}}$ & $122.7^{\mathrm{d}}$ & 112.9 & 133.2 \\
$\mathbf{4}$ & $145.2^{\mathrm{d}}$ & $144.1^{\mathrm{d}}$ & $116.6^{\mathrm{d}}$ & $122.9^{\mathrm{d}}$ & $120.8^{\mathrm{d}}$ & $106.6^{\mathrm{d}}$ & $131.2^{\mathrm{d}}$ \\
$\mathbf{5}$ & 128.3 & 142.7 & 118.4 & 123.1 & $122.6^{\mathrm{d}}$ & 114.0 & 135.2 \\
$\mathbf{3 0}$ & 145.9 & 143.2 & 115.9 & $137.3^{\mathrm{d}}$ & 118.2 & 112.2 & 143.0 \\
$\mathbf{3 1}$ & 146.7 & 143.2 & 120.1 & 117.9 & 132.6 & 108.5 & 148.0 \\
$\mathbf{3 2}$ & 126.5 & 143.2 & 119.1 & $123.0^{\mathrm{e}}$ & $123.0^{\mathrm{e}}$ & 112.0 & 132.9 \\
$\mathbf{3 3}$ & $144.7^{\mathrm{d}}$ & $143.9^{\mathrm{d}}$ & $116.8^{\mathrm{d}}$ & $123.5^{\mathrm{d}}$ & $121.3^{\mathrm{d}}$ & $106.9^{\mathrm{d}}$ & $131.4^{\mathrm{d}}$ \\
$\mathbf{3 5}$ & 133.9 & 142.7 & 118.6 & $122.8^{\mathrm{e}}$ & $122.8^{\mathrm{e}}$ & 133.6 & 135.0 \\
$\mathbf{2 6 b ^ { 3 7 }}$ & 142.2 & 143.8 & 119.6 & 122.7 & 122.1 & 111.4 & 133.0 \\
\hline
\end{tabular}


Table 8. (continued)

\begin{tabular}{llllll}
\hline & C-1' & C-2' & C-3' & C-4' & C-5' \\
\hline $\mathbf{1}$ & 85.0 & ' $^{\text {c }}$ & 70.4 & 87.9 & 61.3 \\
$\mathbf{2}$ & 85.1 & - $^{\text {c }}$ & 70.3 & 87.8 & 61.2 \\
$\mathbf{3}$ & 86.4 & 38.4 & 70.2 & 87.4 & 61.3 \\
$\mathbf{4}$ & 85.7 & 41.8 & 69.1 & 87.8 & 60.5 \\
$\mathbf{5}$ & 86.0 & 97.4 & 73.8 & 82.9 & 59.9 \\
$\mathbf{3 0}$ & 81.8 & 36.5 & 74.6 & 85.2 & 63.9 \\
$\mathbf{3 1}$ & 81.9 & 36.6 & 74.8 & 85.1 & 64.1 \\
$\mathbf{3 2}$ & 80.7 & 35.4 & 73.5 & 85.9 & 63.6 \\
$\mathbf{3 3}$ & 81.9 & 37.8 & 74.3 & 85.1 & 63.7 \\
$\mathbf{3 5}$ & 86.2 & 94.9 & 76.3 & 77.3 & 62.6 \\
$\mathbf{2 6 b}$ & 84.5 & - & 70.6 & 87.6 & 61.6 \\
\hline
\end{tabular}

${ }^{a}$ Measured in DMSO- $\mathrm{d}_{6}$ at $298 \mathrm{~K}$. ${ }^{\mathrm{b}}$ Systematic numbering. ${ }^{\mathrm{c}}$ Superimposed by the DMSO signal. ${ }^{\mathrm{d}}$ Tentative assignment. ${ }^{\mathrm{e}}$ The signals of C5 and C6 are superimposing.

Table 9. $J_{\mathrm{C}, \mathrm{H}}$ and $J_{\mathrm{C}, \mathrm{F}}$ values (in $\mathrm{Hz}$ ) of $\beta$-L- and $\beta$-D-2'-deoxyribonucleosides ${ }^{\mathrm{a}, \mathrm{b}}$

\begin{tabular}{|c|c|c|c|}
\hline & 5 & 32 & 35 \\
\hline $\mathrm{C} 2, \mathrm{H} 2$ & - & - & - \\
\hline $\mathrm{C} 2, \mathrm{H} 1{ }^{\prime}$ & 3.5 & 8.3 & $\mathrm{~m}^{\mathrm{e}}$ \\
\hline $\mathrm{C} 3 \mathrm{a}, \mathrm{H} 4$ & 14.1 & 15.5 & 15.5 \\
\hline C4, H4 & 165.0 & 162.1 & 162.6 \\
\hline C5, H5 & 160.4 & $162.7^{\mathrm{c}}$ & $160.0^{c}$ \\
\hline C6, H6 & 160.1 & $162.7^{\mathrm{c}}$ & $160.0^{c}$ \\
\hline C7, H7 & 168.7 & 165.5 & 168.4 \\
\hline $\mathrm{C} 7 \mathrm{a}, \mathrm{H} 7$ & 20.1 & 23.0 & $\mathrm{~m}^{\mathrm{e}}$ \\
\hline C1'-H1' & 161.0 & 151.2 & 161.5 \\
\hline $\mathrm{C} 2^{\prime}-\mathrm{H} 2^{\prime}$ & 193.5 & 135.8 & 193.8 \\
\hline C3'-H3' & 149.0 & 156.6 & 156.2 \\
\hline C4', H4' & 147.7 & 162.7 & 151.5 \\
\hline C5' $-\mathrm{H} 5^{\prime}$ & 142.4 & 149.5 & 151.7 \\
\hline C1'-F2' & 17.5 & - & 18.0 \\
\hline C2'-F2' & 193.1 & - & 194.1 \\
\hline C3'-F2' & 24.2 & - & 28.8 \\
\hline $\mathrm{C} 4{ }^{\prime}-\mathrm{F} 2^{\prime}$ & 4.8 & - & - \\
\hline
\end{tabular}

${ }^{a}$ Measured in DMSO-d 6 at $298 \mathrm{~K}$. ${ }^{\mathrm{b}}$ Systematic numbering is used. ${ }^{\mathrm{c}}$ The signals of C5 and C6 are superimposing. ${ }^{\mathrm{d}}$ Superimposed by the DMSO signal. ${ }^{\mathrm{e}}$ Superimposing multipletts. 
Furthermore, the solid state structure of the benzoylated 2-bromo-1-[2-deoxy-2-fluoro- $\beta$-Darabinofuranosyl]-benzimidazole 35 was confirmed by single-crystal x-ray analysis. ${ }^{38}$

From the crystal structure of $\mathbf{3 5}$, the orientation of the nucleobase relative to the sugar moiety was determined to be syn with $\chi=\left(\mathrm{O} 1^{\prime}-\mathrm{C} 1{ }^{\prime}-\mathrm{N} 11-\mathrm{C} 18\right)=39.7(9)^{\circ}$. The natural 2'deoxyribonucleosides usually adopt an anti conformation. However, it is observed that the introduction of a bulky substituent in position-2 of the heterocyclic moiety shifts the conformation around the glycosylic bond from anti to syn as it was recently reported for 2'deoxyimmunosine. ${ }^{39}$ The sugar moiety of $\mathbf{3 5}$ shows a pseudorotation phase angle $P$ of $118.8^{\circ}$ and a maximum amplitude of puckering $\tau_{\mathrm{m}}$ of $41.8^{\circ}$ which indicates a south $(S)$ conformation. This result is in contrast to many other 2'-fluoroarabino nucleosides that are in $N$-conformation. However, the conformation of the sugar residue is also strongly influenced by the benzoyl protecting groups.

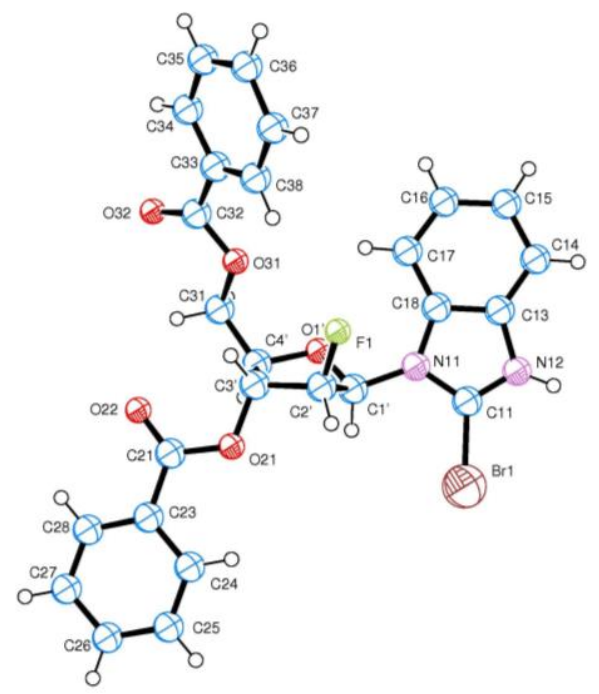

Figure 7. The crystal structure of compound $\mathbf{3 5}$ obtained from single crystal X-ray analysis.

\section{Conclusions}

The antiviral activity of a series of benzimidazole derivatives and substituted benzimidazole $\beta$-L- and $\beta$-D-nucleosides against selected RNA and DNA viruses including HIV-1, BVDV, YFV, DENV-2, WNV, HBV, HCV and human RSV was evaluated. Unfortunately, no selective antiviral activity was observed for the tested compounds. However, among the halogenated benzimidazoles including the 4,5,6,7-tetrabrominated benzimidazoles $\beta$-L-2'deoxyribonucleoside $\mathbf{4}$ several derivatives were found to be cytotoxic against the tested cell lines. 
These compounds are potential candidates for anti-cancer screening.

Furthermore, the stereoselective synthesis of benzimidazole $\beta$-L-2'-deoxyribonucleosides and the 2-bromobenzimidazole 2'-deoxy-2'-fluoro- $\beta$-D-arabinofuranosyl nucleoside was accomplished using nucleobase anion glycosylation. For that, 3,5-di-O-(4-methylbenzoyl)-2deoxy- $\alpha$-L-erythro-pentofuranosyl chloride or 3,5-di- $O$-benzoyl-2-deoxy-2-fluoro- $\alpha$-Darabinofuranosyl bromide were employed as sugar residues. The compounds were characterized by NMR spectroscopy and compound 35 by single-crystal X-ray analysis.

\section{Experimental Section}

General Procedures. All chemicals were purchased from Acros, Fluka or Sigma-Aldrich. Solvents were of technical grade and were distilled before use. Thin-layer chromatography (TLC) was carried out on TLC aluminium sheets covered with silica gel $60 \mathrm{~F}_{254}, 0.2 \mathrm{~mm}$ layer (VWR, Germany). Column flash chromatography (FC) was performed at 0.4 bar on silica gel 60 H (VWR, Germany). UV-spectra: U3200 spectrophotometer (Hitachi, Japan); $\lambda_{\max }$ in nm, $\varepsilon$ in $\mathrm{dm}^{3} \mathrm{~mol}^{-1}$. NMR Spectra: Avance-DPX-250, Avance-DPX-300 or AMX-500 spectrometers (Bruker, Rheinstetten, Germany) at $250.13,300.15$ and $500 \mathrm{MHz}$ for ${ }^{1} \mathrm{H}$ and ${ }^{13} \mathrm{C}$; chemical shifts $(\delta)$ are in $\mathrm{ppm}$ rel. to internal $\mathrm{SiMe}_{4}\left({ }^{1} \mathrm{H},{ }^{13} \mathrm{C}\right)$. Microanalyses were performed by Mikroanalytisches Labor Beller (Göttingen, Germany).

\section{1-[2-Deoxy-3,5-di- $O$-(4-methylbenzoyl)- $\beta$-L-erythro-pentofuranosyl]-5-nitrobenzimidazole (30) and 1-} [2-Deoxy-3,5-di- $\boldsymbol{O}$-(4-methylbenzoyl)- $\boldsymbol{\beta}$-L-erythro-pentofuranosyl]-6-nitrobenzimidazole (31). To a suspension of powdered KOH (1.013 g, $18.0 \mathrm{mmol})$ and TDA-1 (178 mg, $0.6 \mathrm{mmol})$ in anhyd. $\mathrm{CH}_{3} \mathrm{CN}$ (300 ml) 5(6)-nitrobenzimidazole (28; $980 \mathrm{mg}, 6.0 \mathrm{mmol}$ ) was added at room temperature. After the mixture was stirred for $30 \mathrm{~min}, 3,5$-di- $O$-(4-methylbenzoyl)-2-deoxy- $\alpha$-Lerythro-pentofuranosyl chloride $(29 ; 2.45 \mathrm{~g}, 6.3 \mathrm{mmol})$ was introduced and stirring was continued for $120 \mathrm{~min}$. Insoluble material was filtered off and the solvent was evaporated. The residue was dissolved in $\mathrm{CH}_{2} \mathrm{Cl}_{2}$ and purified by flash chromatography (FC) (silica gel, 50 x 5.5 $\mathrm{cm})$ and the compounds $\mathbf{3 0}$ and $\mathbf{3 1}$ were eluted with $\mathrm{CH}_{2} \mathrm{Cl}_{2} / \mathrm{EtOAc}(8: 2)$.

From the faster migrating zone, compound $\mathbf{3 0}$ was isolated. After evaporation of the solvent $\mathbf{3 0}$ was obtained as colorless crystals (0.8 g, 26\%). M.p. 134-135 ${ }^{\circ} \mathrm{C}$. TLC (silica gel, $\mathrm{CH}_{2} \mathrm{Cl}_{2} /$ EtOAc 8:2): $\mathrm{R}_{\mathrm{f}}$ 0.70. UV (MeOH): 238 (49400), 298 (8800). ${ }^{1} \mathrm{H}$ NMR (500 MHz, DMSO-d $\left.d_{6}\right): \delta 2.39,2.42\left(2 \mathrm{~s}, 6 \mathrm{H}, 2 \mathrm{x}-\mathrm{CH}_{3}\right), 2.87\left(\mathrm{~m}, 1 \mathrm{H}, \mathrm{H}-2{ }^{\prime}{ }_{\beta}\right), 3.11\left(\mathrm{~m}, 1 \mathrm{H}, \mathrm{H}-2{ }_{\alpha}{ }_{\alpha}\right), 4.58(\mathrm{~m}$, $3 \mathrm{H}, \mathrm{H}-4$ ', H-5'), 5.75 (d, $J=5.7 \mathrm{~Hz}, 1 \mathrm{H}, \mathrm{H}-3$ '), 6.69 ('t', $J=6.9 \mathrm{~Hz}, 1 \mathrm{H}, \mathrm{H}-1$ '), 7.36 (dd, $J=$ 7.8, $7.9 \mathrm{~Hz}, 4 \mathrm{H}$, arom. H), 8.01 (m, 6H, arom. H, H-6, H-7), 8.56 (s, 1H, H-4), 8.81 (s, 1H, H-2). Anal. Calcd. for $\mathrm{C}_{28} \mathrm{H}_{25} \mathrm{~N}_{3} \mathrm{O}_{7}$ (515.5) requires: C, 65.24; H, 4.89; N, 8.15. Found: C, 65.15; H, $4.87 ; \mathrm{N}, 8.10$.

From the slower migrating zone, compound 31 was isolated as colorless foam (1.0 g, 32\%). M.p. 75-80 ${ }^{\circ} \mathrm{C}$. TLC (silica gel, $\mathrm{CH}_{2} \mathrm{Cl}_{2} / \mathrm{EtOAc}, 8: 2$ ): $\mathrm{R}_{\mathrm{f}}$ 0.62. UV (MeOH): 237 (52900), 296 
(10400). ${ }^{1} \mathrm{H}$ NMR (500 MHz, DMSO-d6): $\delta$ 2.36, $2.42\left(2 \mathrm{~s}, 6 \mathrm{H}, 2 \mathrm{x}-\mathrm{CH}_{3}\right), 2.91\left(\mathrm{~m}, 1 \mathrm{H}, \mathrm{H}-2{ }^{\prime}{ }\right)$, 3.13 (m, 1H, H-2' ${ }_{\alpha}$ ), 4.57 (m, 2H, H-5'), 4.67 (m, 1H, H-4'), 5.73 (d, J = 5.6 Hz, 1H, H-3'), 6.78 ('t', $J=6.8 \mathrm{~Hz}, 1 \mathrm{H}, \mathrm{H}-1$ ') 7.35 (dd, $J=7.8,7.9 \mathrm{~Hz}, 4 \mathrm{H}$, arom. H), 7.85 (m, 5H, arom. H, H-4), $8.15(\mathrm{~d}, J=8.9 \mathrm{~Hz}, 1 \mathrm{H}, \mathrm{H}-5), 8.78$ (s, $1 \mathrm{H}, \mathrm{H}-7), 8.87$ (s, $1 \mathrm{H}, \mathrm{H}-2)$. Anal. Calcd. for $\mathrm{C}_{28} \mathrm{H}_{25} \mathrm{~N}_{3} \mathrm{O}_{7}$ (515.5) requires: $\mathrm{C}, 65.24 ; \mathrm{H}, 4.89 ; \mathrm{N}, 8.15$. Found: $\mathrm{C}, 65.26 ; \mathrm{H}, 4.95 ; \mathrm{N}, 8.11$.

1-[2-Deoxy- $\beta$-L-erythro-pentofuranosyl)-5-nitrobenzimidazole (1). A solution of compound 30 (500 mg, $0.97 \mathrm{mmol})$, dissolved in a mixture of $\mathrm{MeOH}(130 \mathrm{ml})$ and $1 \mathrm{M} \mathrm{NaOMe} / \mathrm{MeOH}$ $(2.0 \mathrm{ml})$ was stirred at room temperature for $48 \mathrm{~h}$. After evaporation of the solvent a solid residue was obtained which was applied to FC (silica gel, column 3 x $20 \mathrm{~cm}, \mathrm{CHCl}_{3} / \mathrm{MeOH}, 85: 15$ ). Crystallisation from $\mathrm{MeOH}$ afforded 1 as colorless crystals (160 mg, 59\%). M.p. 157-160 ${ }^{\circ} \mathrm{C}$. TLC (silica gel, $\left.\mathrm{CHCl}_{3} / \mathrm{MeOH}, 85: 15\right): \mathrm{R}_{\mathrm{f}}$ 0.79. UV (MeOH): 238 (27100), 298 (9200). ${ }^{1} \mathrm{H}-\mathrm{NMR}$ (500 MHz, DMSO- $\left.d_{6}\right): \delta 2.37\left(\mathrm{~m}, 1 \mathrm{H}, \mathrm{H}-2{ }^{\prime}{ }_{\beta}\right), 2.62\left(\mathrm{~m}, 1 \mathrm{H}, \mathrm{H}-2{ }^{\prime}{ }_{\alpha}\right), 3.88$ (m, 2H, H-5'), 3.91 (m, 1H, H-4'), 4.42 (m, 1H, H-3'), 5.03 (m, 1H, 5'-OH), 5.40 (d, J=3.9 Hz, 1H, 3'-OH), 6.47 ('t', $J$ = $\left.6.6 \mathrm{~Hz}, 1 \mathrm{H}, \mathrm{H}-1^{\prime}\right), 7.99$ (d, J = 9.0 Hz, 1H, H-7), 8.19 (d, J=9.1 Hz, 1H, H-6), 8.58 (s, 1H, H4), 8.80 (s, 1H, H-2). Anal. Calcd. for $\mathrm{C}_{12} \mathrm{H}_{13} \mathrm{~N}_{3} \mathrm{O}_{5}$ (279.3) requires: C, 51.61; H, 4.69; N, 15.05. Found: C, 51.59; H, 4.61; N, 14.89 .

1-[2-Deoxy- $\boldsymbol{\beta}$-L-erythro-pentofuranosyl)-6-nitrobenzimidazole (2). As described for $\mathbf{1}$, with compound 31 (600 mg, $1.16 \mathrm{mmol})$, in $\mathrm{MeOH}(20 \mathrm{ml})$ and $1 \mathrm{M} \mathrm{NaOMe} / \mathrm{MeOH}(2.5 \mathrm{ml})$ for $72 \mathrm{~h}$. FC resulted in a colorless solid. Crystallisation from $\mathrm{MeOH}$ afforded 2 as colorless crystals (200 mg, 62\%). M.p. $185-187{ }^{\circ} \mathrm{C}$. TLC (silica gel, $\left.\mathrm{CHCl}_{3} / \mathrm{MeOH}, 8: 2\right): \mathrm{R}_{\mathrm{f}}$ 0.66. UV (MeOH): 234 (16400), 297 (9800). ${ }^{1} \mathrm{H}$ NMR (500 MHz, DMSO-d6): $\delta 2.38$ (m, 1H, H-2' $\left.{ }_{\beta}\right), 2.64$ (m, 1H, H-2' ${ }_{\alpha}$ ), 3.59 (m, 2H, H-5'), 3.92 (m, 1H, H-4'), 4.44 (m, 1H, H-3'), 5.05 (m, 1H, 5'-OH), 5.39 (d, $J=3.9 \mathrm{~Hz}, 1 \mathrm{H}, 3$ '-OH), 6.54 ('t', $J=6.5 \mathrm{~Hz}, 1 \mathrm{H}, \mathrm{H}-1$ ') 7.88 (d, $J=8.9 \mathrm{~Hz}, 1 \mathrm{H}, \mathrm{H}-4), 8.15$ $(\mathrm{d}, J=7.8 \mathrm{~Hz}, 1 \mathrm{H}, \mathrm{H}-5), 8.80$ (s, $1 \mathrm{H}, \mathrm{H}-7), 8.85$ (s, 1H, H-2). Anal. Calcd. for $\mathrm{C}_{12} \mathrm{H}_{13} \mathrm{~N}_{3} \mathrm{O}_{5}$ (279.3) requires: C, 51.61; H, 4.69; N, 15.05. Found: C, 51.60; H, 4.59; N, 14.90.

\section{2-Bromo-1-[2-deoxy-3,5-di-O-(4-methylbenzoyl)- $\beta$-L-erythro-pentofuranosyl]-benzimidazole}

(32).

$\mathrm{NaH}$ (80 mg, $2 \mathrm{mmol}, 60 \%$ in oil) was added portionwise to a suspension of 2bromobenzimidazole (12a; $295.5 \mathrm{mg}, 1.5 \mathrm{mmol})$ in $25 \mathrm{ml}$ anhyd. $\mathrm{MeCN}$ at room temperature. The reaction mixture was stirred for additional $10 \mathrm{~min}$ at $\mathrm{rt}$. To this solution compound 29 $(583.5 \mathrm{mg}, 1.5 \mathrm{mmol}$ ) was added portionwise within $5 \mathrm{~min}$ and stirring was continued for $20 \mathrm{~min}$ at $\mathrm{rt}$. The reaction mixture was diluted with $\mathrm{CH}_{2} \mathrm{Cl}_{2}(20 \mathrm{ml})$, filtered through a Celite pad, and the filtrate was evaporated. The residue was triturated with $\mathrm{MeOH}$. The resulting suspension was filtered and compound 32 was obtained from $\mathrm{MeOH}$ as a white solid (520 mg, 63\%). M.p. 117$11{ }^{\circ} \mathrm{C}$. TLC (silica gel, $\left.\mathrm{CHCl}_{3} / \mathrm{MeOH}, 95: 5\right): \mathrm{R}_{\mathrm{f}}$ 0.78. UV (MeOH): 242 (38800), 275 (8400), 282 (7600). ${ }^{1} \mathrm{H}$ NMR (250 MHz, DMSO-d $): \delta 2.38,2.40\left(2 \mathrm{~s}, 6 \mathrm{H}, 2 \mathrm{x}-\mathrm{CH}_{3}\right), 2.66(\mathrm{~m}, 1 \mathrm{H}, \mathrm{H}-$ 2' $\beta$ ), 3.03 (m, 1H, H-2' $\alpha$ ), 4.62 (m, 1H, H-4'), 4.75 (m, 2H, H-5'), 5.79 (m, 1H, H-3'), 6.53 ('t', J $\left.=7.0 \mathrm{~Hz}, 1 \mathrm{H}, \mathrm{H}-1^{\prime}\right), 6.93(\mathrm{t}, J=7.7 \mathrm{~Hz}, 1 \mathrm{H}, \mathrm{H}-5), 7.22$ (t, $\left.J=7.4 \mathrm{~Hz}, 1 \mathrm{H}, \mathrm{H}-6\right), 7.61$ (d, $J=7.9$ $\mathrm{Hz}, 1 \mathrm{H}, \mathrm{H}-4), 7.70(\mathrm{~d}, J=8.0 \mathrm{~Hz}, 1 \mathrm{H}, \mathrm{H}-7)$ and arom. $\mathrm{H}$ of the protecting groups. Anal. Calcd. for $\mathrm{C}_{28} \mathrm{H}_{25} \mathrm{~N}_{2} \mathrm{BrO}_{5}(549.4)$ requires: $\mathrm{C}, 61.21 ; \mathrm{H}, 4.59 ; \mathrm{N}, 5.10$. Found: $\mathrm{C}, 61.12 ; \mathrm{H}, 4.50 ; \mathrm{N}$, 5.11 . 
2-Bromo-1-[2-deoxy- $\boldsymbol{\beta}$-L-erythro-pentofuranosyl]-benzimidazole (3). A solution of compound 32 (420 mg, $0.76 \mathrm{mmol})$, dissolved in a mixture of $\mathrm{MeOH}(15 \mathrm{ml})$ and $1 \mathrm{M} \mathrm{NaOMe} / \mathrm{MeOH}$ $(2 \mathrm{ml})$ was stirred for $1 \mathrm{~h}$ at $\mathrm{rt}$. After evaporation of the solvent a solid residue was obtained which was applied to FC (silica gel, column $2 \times 20 \mathrm{~cm}, \mathrm{CH}_{2} \mathrm{Cl}_{2} / \mathrm{MeOH}, 9: 1$ ). Evaporation of the solvent and crystallization from EtOH gave compound $\mathbf{3}$ as a colorless solid (210 $\mathrm{mg}, 88 \%$ ). M.p. $150-152{ }^{\circ} \mathrm{C}$. TLC (silica gel, $\mathrm{CH}_{2} \mathrm{Cl}_{2} / \mathrm{MeOH}, 9: 1$ ): $\mathrm{R}_{\mathrm{f}}$ 0.69. UV (MeOH): 246 (7900), 267 (5100), 275 (6400), 282 (6200). ${ }^{1} \mathrm{H}$ NMR (250 MHz, DMSO- $\left.d_{6}\right): \delta 2.17$ (m, 1H, H-2' $\left.{ }_{\beta}\right), 2.62(\mathrm{~m}$, 1H, H-2' ${ }_{\alpha}$ ), 3.68 (m, 2H, H-5'), 3.88 (m, 1H, H-4'), 4.43 (m, 1H, H-3'), 5.08 (m, 1H, 5'-OH), 5.44 (d, $\left.J=3.1 \mathrm{~Hz}, 1 \mathrm{H}, 3^{\prime}-\mathrm{OH}\right), 6.37$ (dd, $J=6.1,6.0 \mathrm{~Hz}, 1 \mathrm{H}, \mathrm{H}-1$ '), 7.25 (m, 2H, H-4, H-7), 7.63 (m, 1H, H-5), 7.93 (m, 1H, H-6). Anal. Calcd. for $\mathrm{C}_{12} \mathrm{H}_{13} \mathrm{~N}_{2} \mathrm{BrO}_{3}$ (313.1) requires: C, 46.03; H, 4.18; N, 8.95. Found: C, 45.94; H, 4.16; N, 8.78.

\section{1-[2-Deoxy-3,5-di- $O$-(4-methylbenzoyl)- $\beta$-L-erythro-pentofuranosyl]-4,5,6,7-}

tetrabromobenzimidazole (33). $\mathrm{NaH}(80 \mathrm{mg}, 2 \mathrm{mmol}, 60 \%$ in oil) was added portionwise to a suspension of 4,5,6,7-tetrabromobenzimidazole (6a; $651 \mathrm{mg}, 1.5 \mathrm{mmol})$ in anhyd. $\mathrm{MeCN}(25 \mathrm{ml})$ at room temperature. The reaction mixture was stirred for additional $10 \mathrm{~min}$ at $\mathrm{rt}$ and then heated under reflux for $10 \mathrm{~min}$. Compound $29(583.5 \mathrm{mg}, 1.5 \mathrm{mmol})$ was added, and stirring was continued for $20 \mathrm{~min}$ at $\mathrm{rt}$. The reaction mixture was diluted with $\mathrm{CH}_{2} \mathrm{Cl}_{2}(20 \mathrm{ml})$, filtered through a Celite pad, and the filtrate was evaporated. The dry material was applied onto FC (silica gel, column $3.5 \times 20 \mathrm{~cm}, \mathrm{CH}_{2} \mathrm{Cl}_{2}$ ). Compound 33 was obtained from $\mathrm{MeOH}$ as a white solid (600 mg, 51\%). M.p. 191-193 ${ }^{\circ} \mathrm{C}$. TLC (silica gel, petroleum ether/ethyl acetate, 2:1): $\mathrm{R}_{\mathrm{f}}$ 0.63. UV (MeOH): 230 (27500). ${ }^{1} \mathrm{H}$ NMR (250 MHz, DMSO-d $): \delta 2.38,2.41(2 \mathrm{~s}, 6 \mathrm{H}, 2 \mathrm{x}-$ $\mathrm{CH}_{3}$ ), 2.97 (m, 1H, H-2' $\left.{ }^{\prime}\right), 3.13$ (m, 1H, H-2' ${ }_{\alpha}$ ), 4.53 (m, 1H, H-4'), 4.57, 4.60 (2m, 2H, H-5'), 5.71 (m, 1H, H-3'), 7.16 (' $\mathrm{t}$ ', $J=6.4,1 \mathrm{H}, \mathrm{H}-1$ '), 8.83 (s, 1H, H-2) and $\mathrm{H}$ arom. of the protecting groups. Anal. Calcd. for $\mathrm{C}_{28} \mathrm{H}_{22} \mathrm{~N}_{2} \mathrm{Br}_{4} \mathrm{O}_{5}$ (786.1) requires: C, 42.78; H, 2.82; N, 3.56. Found: C, 42.69; H, 2.74; N, 3.58.

1-[2-Deoxy- $\boldsymbol{\beta}$-L-erythro-pentofuranosyl]-4,5,6,7-tetrabromobenzimidazole (4). A solution of compound 33 (500 mg, $0.64 \mathrm{mmol}$ ) in $\mathrm{MeOH}(20 \mathrm{ml})$ and $1 \mathrm{M} \mathrm{NaOMe} / \mathrm{MeOH}$ (2.5 ml) was stirred under reflux for $10 \mathrm{~min}$. Silica gel 60 (2 g) was added, and the solvent was evaporated. The dry material was applied onto $\mathrm{FC}$ (silica gel, column 2 x $10 \mathrm{~cm}, \mathrm{CHCl}_{3} / \mathrm{MeOH}, 9: 1$ ). Compound 4 was obtained from $\mathrm{MeOH}$ as a white solid $(180 \mathrm{mg}, 51 \%)$. M.p. 92-96 ${ }^{\circ} \mathrm{C}$. TLC (silica gel, $\left.\mathrm{CHCl}_{3} / \mathrm{MeOH}, 9: 1\right): \mathrm{R}_{\mathrm{f}}$ 0.39. UV (MeOH): 268 (10700). ${ }^{1} \mathrm{H}$ NMR (250 MHz, DMSO-d $\left.d_{6}\right): \delta 2.48$ (m, 1H, H-2 ' $\left.{ }_{\beta}\right), 2.57$ (m, 1H, H-2' ${ }_{\alpha}$ ), 3.59, 3.63 (2m, 2H, H-5'), 3.89 (m, 1H, H-4'), 4.37 (m, 1H, H-3'), 5.10 (m, 1H, 5'-OH), 5.39 (m, 1H, 3'-OH), 7.01 (m, 1H, H-1'), 8.89 (s, 1H, H-2). Anal. Calcd. for $\mathrm{C}_{12} \mathrm{H}_{10} \mathrm{~N}_{2} \mathrm{Br}_{4} \mathrm{O}_{3}$ (549.8) requires: C, 26.21; H, 1.83; N, 5.09. Found: C, 26.25; H, 2.00; N, 5.16.

\section{2-Bromo-1-[2-deoxy-2-fluoro-3,5-di- $O$-benzoyl- $\beta$-D-arabinofuranosyl]-benzimidazole (35).} Compound 12a (230 mg, $1.2 \mathrm{mmol}$ ) was dissolved in $20 \mathrm{ml}$ anhyd. MeCN. After addition of powdered $\mathrm{KOH}(200 \mathrm{mg}, 3.6 \mathrm{mmol}$ ), the solution was stirred for $15 \mathrm{~min}$ at $\mathrm{rt}$. Then TDA-1 (70 mg, $0.22 \mathrm{mmol}$ ) was added and stirring was continued for another $15 \mathrm{~min}$ at rt. After addition of the fluoro sugar 34 (400 mg, $0.945 \mathrm{mmol})$ in $\mathrm{MeCN}(5 \mathrm{ml})$, stirring was continued for 
additional $1.5 \mathrm{~h}$. Insoluble material was filtered off and the filtrate was evaporated to dryness (water bath temperature of the rotation evaporator not exceeding $35^{\circ} \mathrm{C}$ ). The resulting syrup was absorbed on silica gel and applied to FC (silica gel, column $2.5 \times 20 \mathrm{~cm}$, petroleum ether/ethyl acetate, 3:1). Compound 35 was obtained from $\mathrm{MeOH}$ as colorless crystals (270 $\mathrm{mg}, 42 \%$ ). TLC (silica gel, petroleum ether/ethyl acetate, 1:1): $\mathrm{R}_{\mathrm{f}}=0.70$. M.p. $60-63{ }^{\circ} \mathrm{C}$. UV $(\mathrm{MeOH}): 267$ (5200), 273 (6300), 281 (5700). ${ }^{1} \mathrm{H}-\mathrm{NMR}$ (300 MHz, DMSO-d6): $\delta 4.74$ (m, 2H, H-5'), 4.91 (m, $1 \mathrm{H}, \mathrm{H}-4^{\prime}$ ), 5.76 (dd, $J_{2}{ }^{\prime}, \mathrm{F}=32.5 \mathrm{~Hz}, 1 \mathrm{H}, \mathrm{H}-2^{\prime}$ ), 5.89 (m, 1H, H-3'), 6.66 (dd, $J_{1^{\prime}, \mathrm{F}}=20.9 \mathrm{~Hz}, J_{1^{\prime}, 2^{\prime}}$ $=3.8 \mathrm{~Hz}, 1 \mathrm{H}, \mathrm{H}-1$ ') $, 6.82(\mathrm{t}, J=7.7 \mathrm{~Hz}, 1 \mathrm{H}, \mathrm{H}-5), 7.15$ (t, $J=7.7 \mathrm{~Hz}, 1 \mathrm{H}, \mathrm{H}-6), 7.7,8.09$ (2m, $12 \mathrm{H}, \mathrm{H}-4, \mathrm{H}-7$ and arom. $\mathrm{H}$ of the protecting groups). Anal. Calcd. for $\mathrm{C}_{26} \mathrm{H}_{20} \mathrm{~N}_{2} \mathrm{FBrO}_{5}(539.35)$ requires: $\mathrm{C}, 57.90 ; \mathrm{H}, 3.74 ; \mathrm{N}, 5.19$. Found: $\mathrm{C}, 57.98 ; \mathrm{H}, 3.81 ; \mathrm{N}, 5.25$.

2-Bromo-1-[2-deoxy-2-fluoro- $\boldsymbol{\beta}$-D-arabinofuranosyl]-benzimidazole (5). Compound 35 (240 mg, $0.44 \mathrm{mmol}$ ) was dissolved in methanolic ammonia (saturated at $0{ }^{\circ} \mathrm{C}, 50 \mathrm{ml}$ ) and the soution was stirred at room temperature for $5 \mathrm{~h}$. The solvent was evaporated and the residue applied to FC (silica gel, column $2 \times 10 \mathrm{~cm}, \mathrm{CH}_{2} \mathrm{Cl}_{2} / \mathrm{MeOH}, 95: 5$ ). The product containing fractions were collected and the solvent was evaporated to dryness to give compound $\mathbf{5}$ as a white solid (90 mg, 61\%). TLC (silica gel, $\mathrm{CH}_{2} \mathrm{Cl}_{2} / \mathrm{MeOH}, 9: 1$ ): $\mathrm{R}_{\mathrm{f}}=0.84$. M.p. $177-179{ }^{\circ} \mathrm{C}$. UV (MeOH): 245 (5700), 266 (4000), 273 (5000), 281 (4800). ${ }^{1} \mathrm{H}-\mathrm{NMR}$ (300 MHz, DMSO-d6): $\delta$ 3.77 (m, 3H, H-5', H-4'), 4.40 (dd, $J_{3}{ }^{\prime}, \mathrm{F}=23.5 \mathrm{~Hz}, 1 \mathrm{H}, \mathrm{H}-3^{\prime}$ '), 5.10 (t, J=3.1 Hz, 1H, H-2'), 5.28 $\left(\mathrm{t}, J=3.2 \mathrm{~Hz}, 1 \mathrm{H}, 5^{\prime}-\mathrm{OH}\right), 6.01\left(\mathrm{~m}, 1 \mathrm{H}, 3^{\prime}-\mathrm{OH}\right), 6.40\left(\mathrm{dd}, J_{1}\right.$ ', F $=19.3 \mathrm{~Hz}, J_{1}{ }^{\prime}{ }^{\prime}=3.7 \mathrm{~Hz}, 1 \mathrm{H}, \mathrm{H}-$ 1'), 7.22 (m, 2H, H-4, H-7), 7.60 (m, 1H, H-5), 7.85 (m, 1H, H-6). Anal. Calcd. for $\mathrm{C}_{12} \mathrm{H}_{12} \mathrm{~N}_{2}$ $\mathrm{FbrO}_{3}(331.14)$ requires: C, 43.53; H, 3.65; N, 8.46. Found: C, 43.49; H, 3.64; N, 8.44.

\section{Acknowledgements}

The syntheses of the benzimidazole $\beta$-L- and $\beta$-D-nucleosides were performed in the Laboratorium für Organische und Bioorganische Chemie, Institut für Chemie, Universität Osnabrück, Barbarastraße 7, 49069 Osnabrück, Germany. Several antiviral evaluations were carried out by Dr. Michel Liuggi (Idenix Senior Director Virology, Laboratorio Cooperativo Idenix-Universita di Caliari, Italy). His contribution is gratefully acknowledged. The work was supported in part by the Ministry of Science and Higher Education of Poland (PBZ-MIN 014/P05/2004) (ZK) and by Idenix Pharmaceuticals (FS). We thank Dr. Peter Leonard for critical reading the manuscript.

\section{References and Notes}

1. Tunçbilek, M.; Göker, H.; Ertan, R.; Eryigit, R.; Kendi, E.; Altanlar, E. Arch. Pharm. 1997, 330, 372.

2. Preston, P. N. Chem. Rev. 1974, 74, 279 and references citied therein. 
3. Burton, D. E.; Lambie, A. J.; Ludgate, J. C. L.; Newbold, G. T.; Percival, A.; Saggers, D. T. Nature (London) 1965, 208, 1166.

4. Navarrete-Vázquez, G.; Cedillo, R.; Hernández-Campos, A.; Yépez, L.; Hernández-Luis, F.; Valdez, J.; Morales, R.; Cortés, R.; Hernández, M.; Castillo, R. Bioorg. Med. Chem. Lett. 2001, 11, 187.

5. Andrzejewska, M.; Yépez-Mulia, L.; Cedillo-Rivera, R.; Tapia, A.; Vilpo, L.; Vilpo, J.; Kazimierczuk, Z. Eur. J. Med. Chem. 2002, 37, 973.

6. Kazimierczuk, Z.; Andrzejewska, M.; Kaustova, J.; Klimešova, V. Eur. J. Med. Chem. 2005, 40, 203.

7. Stefańska, J. Z.; Gralewska, R.; Starościak, B. J.; Kazimierczuk, Z. Pharmazie 1999, 54, 879.

8. Townsend, L. B.; Devivar, R. V.; Turk, S. R.; Nassiri, M. R.; Drach, J. C. J. Med. Chem. 1995, 38, 4098.

9. North, T. W.; Sequar, G.; Townsend, L. B.; Drach, J. C.; Barry, P. A. Antimicrob. Agents Chemother. 2004, 48, 2760.

10. Townsend, L. B.; Revankar, G. R. Chem. Rev. 1970, 70, 389.

11. (a) Tamm, I. Science 1957, 126, 1235. (b) Sehgal, P. B.; Fraser, N. W.; Darnell, J. E. Virology 1979, 94, 185.

12. Tamm, I.; Kikuchi, T.; Darnell, Jr., J. E.; Salditt-Georgieff, M. Biochemistry 1980, 19, 2743.

13. Tamm, I.; Sehgal, P. B. Adv. Virus Res. 1978, 22, 187.

14. Bucknall, R. A. J. Gen. Virol. 1967, 1, 89.

15. Biron, K. K.; Harvey, R. J.; Chamberlain, S. C.; Good, S. S.; Smith III, A. A.; Davis, M. G.; Talarico, C. L.; Miller, W. H.; Ferris, R.; Dornsife, R. E.; Stanat, S. C.; Drach, J. C.; Townsend, L. B.; Koszalka, G. W. Antimicrob. Agents Chemother. 2002, 46, 2365.

16. Gumina, G.; Chong, Y.; Choo, H.; Song, G.-Y.; Chu, C. K. Curr. Top. Med. Chem. 2002, 2, 1065.

17. Gumina, G.; Song, G.-Y.; Chu, C. K. FEMS Microb. Lett. 2001, 202, 9.

18. Wang, P.; Hong, J. H.; Cooperwood, J. S.; Chu, C. K. Antiviral Res. 1998, 40, 19.

19. Bryant, M. L.; Bridges, E. G.; Placidi, L.; Faraj, A.; Loi, A.-G.; Pierra, C.; Dukhan, D.; Gosselin, G.; Imbach, J.-L.; Hernandez, B.; Juodawlkis, A.; Tennant, B.; Korba, B.; Cote, P.; Marion, P.; Cretton-Scott, E.; Schinazi, R. F.; Sommadossi, J.-P. Antimicrob. Agents Chemother. 2001, 45, 229.

20. Mathé, C.; Gosselin, G. Antiviral Res. 2006, 71, 276.

21. (a) Šmejkal, J.; Šorm, F. Collect. Czech. Commun. 1964, 29, 2809. (b) Gosselin, G.; Pierra, C.; Benzaria-Prad, S.; Dukhan, D.; Cretton-Scott, E.; Standring, D.; Sommadossi, J.-P. Collection Symposium Series (M. Hocek, Ed.), Vol. 10, p. 244. Institute of Organic Chemistry and Biochemistry, Academy of Sciences of the Czech Republic, Prague 2008.

22. Acton, E. M.; Ryan, K. J.; Goodman, L. J. Am. Chem. Soc. 1964, 86, 5352.

23. Baczynski, W.; Niementowski, S. Chem. Zentralbl. 1902, 73, 940.

24. Büchel, K.-H. Z. Naturforsch. 1970, $25 b, 934$.

25. (a) Andrzejewska, M.; Pagano, M. A.; Meggio, F.; Brunati, A. M.; Kazimierczuk, Z. Bioorg. 
Med. Chem. 2003, 11, 3997. (b) Pagano, M. A.; Andrzejewska, M.; Ruzzene, M.; Sarno, S.; Cesaro, L.; Bain, J.; Elliott, M.; Meggio, F.; Kazimierczuk, Z.; Pinna, L. A. J. Med. Chem. 2004, 47, 6239.

26. Andrzejewska, M.; Yepez-Mulia, L.; Tapia, A.; Cedillo-Rivera, R.; Laudy, A. E.; Starościak, B. J.; Kazimierczuk, Z. Eur. J. Pharm. Sci. 2004, 21, 323.

27. Xiangming, H.; Huiqiang, M.; Yulu, W. ARKIVOC 2007, 13, 150.

28. Benzaria, S.; Bardiot, D.; Bouisset, T.; Counor, C.; Rabeson, C.; Pierra, C.; Storer, R.; Loi, A. G.; Cadeddu, A.; Mura, M.; Musiu, C.; Liuzzi, M.; Loddo, R.; Bergelson, S.; Bichko, V.; Bridges, E.; Cretton-Scott, E.; Mao, J.; Sommadossi, J.-P.; Seifer, M.; Standring, D.; Tausek, M.; Gosselin, G.; La Colla, P. Antiviral Chem. Chemother. 2007, 18, 225.

29. Seela, F.; Lin, W.; Kazimierczuk, Z.; Rosemeyer, H.; Glaçon, V.; Peng, X.; He, Y.; Ming, X.; Andrzejewska, M.; Gorska, A.; Zhang, X.; Eickmeier, H.; La Colla, P. Nucleosides Nucleotides Nucleic Acids 2005, 24, 859.

30. (a) Seela, F.; Peng, X. In Current Protocols in Nucleic Acid Chemistry; Beaucage, S. L.; Bergstrom, D. E.; Glick, G. D.; Jones, R. A.; Harkins, E. W.; Eds. 2005, 1.10.1-1.10.20. John Wiley \& Sons, Inc., USA. (b) Seela, F.; Peng, X. Collect. Czech. Chem. Commun. 2006, 71, 956.

31. Hoffer M. Chem. Ber. 1960, 93, 2777.

32. Rabinowitz, J. L.; Wagner, E. C. J. Am. Chem. Soc. 1951, 73, 3030.

33. (a) Winkeler, H.-D.; Seela, F. J. Org. Chem. 1983, 48, 3119. (b) Seela, F.; Westermann, B.; Bindig, U. J. Chem. Soc., Perkin Trans. 1 1988, 697.

34. (a) Seela, F.; Chittepu, P. Org. Biomol. Chem. 2008, 6, 596. (b) Pankiewicz, K. W. Carbohydr. Res. 2000, 327, 87. (c) Watanabe, K. A. Collection Symposium Series 2002, 5, 48.

35. (a) Howell, H. G.; Brodfuehrer, P. R.; Brundidge, S. P.; Benigni, D. A.; Sapino, Jr., C., J. Org. Chem. 1988, 53, 85. (b) Gudmundsson, K. S.; Freeman, G. A.; Drach, J. C.; Townsend, L. B. J. Med. Chem. 2000, 43, 2473. (c) Tann, C. H.; Brodfuehrer, P. R.; Brundidge, S. P.; Sapino, Jr., C., Howell, H. G. J. Org. Chem. 1985, 50, 3644.

36. Seela, F.; Bourgeois, W. Synthesis 1989, 912.

37. Seela, F.; Bourgeois, W.; Rosemeyer, H.; Wenzel, T. Helv. Chim. Acta 1996, 79, 488.

38. Crystal data: $\mathrm{C}_{26} \mathrm{H}_{20} \mathrm{~N}_{2} \mathrm{FBrO}_{5}, \mathrm{MW}=539.36, T=293 \mathrm{~K}, \lambda=0.71073 \AA$, orthorhombic, space group P2 (1) 2 (1) 2 (1), $a=8.535$ (3) $\AA, b=14.165$ (2) $\AA, c=21.546$ (3) $\AA, V=$ 2604.8 (9) $\AA^{3}, Z=4, D=0.362 \mathrm{Mg} / \mathrm{m}^{3}, \mu=0.407 \mathrm{~mm}^{-1}, F(000)=289$. CCDC 698585 contains the supplementary crystallographic data for this paper. These data can be obtained free of charge via www.ccdc.cam.ac.uk/data_request/cif, or by emailing data_request@ccdc.cam.ac.uk, or by contacting The Cambridge Crystallographic Data Centre, 12, Union Road, Cambridge CB2 1 EZ, UK; fax: +44 1223336033.

39. Seela, F.; Ming, X.; Budow, S.; Eickmeier, H.; Reuter, H. Acta Cryst. 2007, C63, o549. 